

\section{QUANTUM STABILIZER CODES}

by

REZA DASTBASTEH

Submitted to the Graduate School of Engineering and Natural Sciences in partial fulfillment of

the requirements for the degree of

Master of Science

Sabancı University

May 2017 


\section{QUANTUM STABILIZER CODES}

APPROVED BY

Prof. Dr. Cem Güneri

(Thesis Supervisor)

Assoc. Prof. Dr. Kağan Kurşungöz

Assist. Prof. Dr. Seher Tutdere

DATE OF APPROVAL: 
(C)Reza Dastbasteh 2017

All Rights Reserved 


\title{
QUANTUM STABILIZER CODES
}

\author{
Reza Dastbasteh
}

Mathematics, Master Thesis, May 2017

Thesis Supervisor: Prof. Dr. Cem Güneri

Keywords: Quantum stabilizer codes, additive codes, self-orthogonal codes, two-dimensional cyclic codes.

\begin{abstract}
We study quantum stabilizer codes and their connection to classical block codes. In addition, different constructions of quantum stabilizer codes and methods of modifying them are presented. Two-dimensional cyclic codes are recalled and a new method of obtaining quantum codes from 2-D cyclic codes is given. We also present a method of obtaining quantum stabilizer codes using additive codes over $\mathbb{F}_{4}$.
\end{abstract}


KUANTUM SABITLEYEN KODLAR

\author{
Reza Dastbasteh \\ Matematik, Yüksek Lisans Tezi, Mayıs 2017 \\ Tez Danışmanı: Prof. Dr. Cem Güneri
} Anahtar Kelimeler: Kuantum sabitleyen kodlar, toplamsal kodlar, kendine dik kodlar,
iki boyutlu devirsel kodlar.

\title{
Özet
}

$\mathrm{Bu}$ tezde kuantum sabitleyen kodlar ve klasik blok kodlarla ilişkileri çalışılmıştır. Çeşitli kuantum sabitleyen kod inşaları ve bu kodları dönüştürme metotları sunulmuştur. İki boyutlu devirsel kodlar yoluyla kuantum sabitleyen kodlar inşa edilmiş, bunun yanı sıra $\mathbb{F}_{4}$ üzerinde toplamsal kodlar kullanılarak kuantum sabitleyen kod inşası sunulmuştur. 
To My Family 


\section{Acknowledgments}

This thesis would not have been possible without the support of many people. First and foremost I would like to express my deepest gratitude to my advisor Dr. Cem Güneri whose guidance, support and assistance from the initial to the final phase has enabled me to successfully develop this thesis.

I would like to thank my committee members, Dr. Kağan Kurşungöz and Dr. Seher Tutdere who have offered their assistance throughout this period.

Finally, I would like to thank my parents, who were not here with me throughout my studies, but they motivated me to pursue the master degree and I constantly received their love and support.

Last but not the least, I would like to thank my wife, Zohreh for her love and unyielding support. Thank you god for giving me all the strength that I needed. 


\section{Table of Contents}

Abstract $\quad$ v

Özet vi

Acknowledgments viii

\begin{tabular}{lll}
\hline & Introduction & 1
\end{tabular}

$1.1 \quad$ History and Overview . . . . . . . . . . . . . . . . . . . . . . . 1

1.2 Cyclic Codes . . . . . . . . . . . . . . . . . . . 2

1.3 Two Dimensional (2-D) Cyclic Codes . . . . . . . . . . . . . . . . . . . 3

1.4 Hermitian Dual of 2-D Cyclic Codes . . . . . . . . . . . . . . . . . . . . . . . 4

1.5 Characterization of 2-D Cyclic Codes . . . . . . . . . . . . . . . . . . . 7

2 Quantum Error Correction Codes 9

2.1 Binary Stabilizer Quantum Codes . . . . . . . . . . . . . . . . . . . . 9 9

2.2 General Constructions of Quantum Stabilizer Codes . . . . . . . . . . . 10

$2.3 \quad$ Quantum Stabilizer Codes from Nearly Self-orthogonal Quaternary Lin-

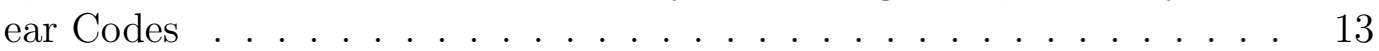

2.4 Non-binary Quantum Stabilizer Codes . . . . . . . . . . . . . . . 16

\begin{tabular}{|lll}
3 & New Constructions of Quantum Stabilizer Codes & 20
\end{tabular}

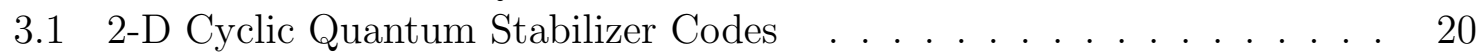

3.2 New Quantum Stabilizer Codes Construction Using 2-D Cyclic Codes . 21

3.3 General Quantum Stabilizer Codes Construction Using Classical Additive Codes . . . . . . . . . . . . . . . . 24

$\begin{array}{ll}\text { Bibliography } & 27\end{array}$ 


\section{CHAPTER 1}

\section{Introduction}

\subsection{History and Overview}

The correspondence between quantum codes and the classical code is a topic which has been studied during the past two decades. There are some similarities between quantum codes and classical codes and we can find a construction for quantum codes by using classical codes. As well as similarities, there are also some substantial differences. [6, 16] are good references that describe the physical and information theoretic motivations behind quantum codes.

In 1995, Shor showed the existence of quantum error-correcting codes [12]. Then, Calderbank and Shor showed that quantum codes can be obtained by using selforthogonal classical quaternary codes [2]. Around the same time, Steane in [14, 15] discovered the existence of good quantum codes with a similar construction. In 1998, Calderbank et al. proposed the exact relation and introduced a wide range of constructions of quantum codes by using classical codes over $\mathbb{F}_{4}[3]$. At the same time, Gottesman independently studied the quantum codes in his $\mathrm{Ph} . \mathrm{D}$. thesis and presented some new ideas of constructing quantum codes [7]. In 2001, Ashikhmin and Knill extended the construction and introduced non-binary quantum stabilizer codes (quantum codes obtained from classical self-orthogonal codes) [1]. The motivation for studying quantum stabilizer codes is due to available simple encoding and decoding algorithms. Finally, Lisonek and Singh proposed a slightly different method of constructing quantum stabilizer codes from linear codes over $\mathbb{F}_{4}$ in $2014[10]$.

In this work, we only consider the quantum stabilizer codes which can be constructed from classical codes. We mostly use [1,3, 10. This thesis is organized as follows:

In Section 1.2, we briefly review cyclic codes and the connection between cyclic codes and polynomial rings. Section 1.3 is concerned with 2-D cyclic codes, a generalization of cyclic codes. We study the connection of 2-D cyclic codes with the polynomial rings, how to find zeros of a 2-D cyclic codes, and a method of finding the Euclidean dual of a 2-D cyclic codes. In section 1.4, we investigate the Hermitian dual of 2-D cyclic codes. Then, in Section 1.5, we give a characterization of all 2-D cyclic codes. Our contribution in this chapter is Section 1.4. The main references of this chapter are 4, 9,11.

In chapter 2 , we study the connection between quantum stabilizer codes and selforthogonal classical codes. The definition of binary stabilizer codes is presented in Section 2.1. Next, we introduce some general methods of modifying and constructing 
binary quantum stabilizer codes in Section 2.2. In Section 2.3, we explain a method of obtaining quantum codes from an arbitrary linear code over $\mathbb{F}_{4}$. Finally, in the last section, we generalize the quantum stabilizer codes construction to the non-binary case, and propose a method of constructing non-binary quantum stabilizer codes. Our main references of this chapter are [1, 3, 5, 10].

In Chapter 3, we present some new constructions of quantum stabilizer codes. For example, we define 2-D cyclic quantum stabilizer codes and characterize all the 2-D cyclic quantum codes in Section 3.1. In Section 3.2, a new method of obtaining quantum codes from 2-D cyclic codes is proposed. Finally, we present a general method of constructing quantum stabilizer codes (both binary and non-binary) from any additive code.

\subsection{Cyclic Codes}

Let $\mathbb{F}_{q}$ be a finite field with characteristic $p$. A $q$-ary linear code $C$ of length $n$ and dimension $k$ is a $k$-dimensional subspace of $\mathbb{F}_{q}^{n}$. Each element of a linear code $C$ is called a codeword. The weight of the codeword $v \in C$ is defined as the number of nonzero coordinates of $v$. The minimum nonzero weight of code $C$ is called the minimum distance of $C$. We denote a linear code of length $n$, dimension $k$, and minimum distance $d$ as $[n, k, d]$ code. Finally, the set of elements in $\mathbb{F}_{q}^{n}$ which are orthogonal to all members of $C$, with respect to the usual (Euclidean) inner product on $\mathbb{F}_{q}^{n}$, is called the dual of the code $C$ and is dented by $C^{\perp}$.

Definition 1.2.1 A linear code is called cyclic if for every $c=\left(c_{0}, c_{1}, \cdots, c_{n-1}\right) \in C$, $\left(c_{n-1}, c_{0}, \cdots, c_{n-2}\right)$ is also in $C$.

In other words, a linear code which is closed under cyclic shift is called cyclic. Obviously, dual of a cyclic code is also a cyclic code. We assume $(n, p)=1$.

Note that the following map is an $\mathbb{F}_{q}$-vector space isomorphism:

$$
\begin{gathered}
\phi: \mathbb{F}_{q}^{n} \longrightarrow \mathbb{F}_{q}[x] /<x^{n}-1> \\
\phi\left(\left(a_{0}, a_{1}, \cdots, a_{n-1}\right)\right) \longmapsto \sum_{i=0}^{n-1} a_{i} x^{i} .
\end{gathered}
$$

Now, we have the following useful criteria for cyclic codes.

Proposition 1.2.1 A linear code $C$ in $\mathbb{F}_{q}^{n}$ is cyclic if and only if $\phi(C)$ is an ideal of $\mathbb{F}_{q}[x] /<x^{n}-1>$.

Proof: Being closed under cyclic shift in $\mathbb{F}_{q}^{n}$ is equivalent to being closed under multiplication by $x$ in $\mathbb{F}_{q}[x] /<x^{n}-1>$.

Note that the ring $\mathbb{F}_{q}[x] /\left\langle x^{n}-1\right\rangle$ is a principal ideal ring, so its ideals can be generated by a single polynomial. The generator polynomial of each ideal, which is a unique monic polynomial of the lowest degree, is called the generator polynomial of the cyclic code. If $g(x)$ is the generator polynomial of the code $C$, then $\operatorname{dim}(C)=$ $n-\operatorname{deg}(g(x))$.

Remark 1.2.1 Let $g(x)$ be the generator polynomial of the code $C$. Then the roots of $g(x)$ in extensions of $\mathbb{F}_{q}$ are called zeros of the code $C$, which are the common zeros of all codewords. The assumption $(n, p)=1$ guarantees that $x^{n}-1$, hence $g(x)$, is separable. Hence, the number of zeros is equal to the degree of $g(x)$. 


\subsection{Two Dimensional (2-D) Cyclic Codes}

Consider the set

$$
\mathbb{F}_{q}^{n_{1} \times n_{2}}=\left\{\left[\begin{array}{ccccc}
a_{0,0} & a_{0,1} & a_{0,2} & \cdots & a_{0, n_{2}-1} \\
a_{1,0} & a_{1,1} & a_{1,2} & \cdots & a_{1, n_{2}-1} \\
\vdots & \vdots & \vdots & \cdots & \ddots \\
a_{n_{1}-1,0} & a_{n_{1}-1,1} & a_{n_{1}-1,2} & \cdots & a_{n_{1}-1, n_{2}-1}
\end{array}\right] \mid a_{i, j} \in \mathbb{F}_{q}\right\},
$$

where $n_{1}$ and $n_{2}$ are positive integers. This set is an $n_{1} n_{2}$-dimensional vector space over $\mathbb{F}_{q}$.

Definition 1.3.2 A linear code $C \subseteq \mathbb{F}_{q}{ }^{n_{1} \times n_{2}}$ is called a 2-D cyclic code of area $n_{1} \times n_{2}$ if $\left(a_{i+s, j+t}\right)$ is also in $C$ for all $s$ and $t$, where $i+s$ and $j+t$ are taken modulo $n_{1}$ and $n_{2}$, respectively.

In other words, a two dimensional (2-D) cyclic code of area $n_{1} \times n_{2}$ is an $\mathbb{F}_{q}$ linear code $C \subseteq \mathbb{F}_{q}{ }^{n_{1} \times n_{2}}$ where $C$ is closed under both column and row shifts. Note that similar to the cyclic case, the dual of a 2-D cyclic code is a 2-D cyclic code and also we have an alternative representation for 2-D cyclic codes. Consider the following $\mathbb{F}_{q}$-vector space isomorphism:

$$
\begin{gathered}
\phi: \mathbb{F}_{q}^{n_{1} \times n_{2}} \longrightarrow \mathbb{F}_{q}[x, y] /<x^{n_{1}}-1, y^{n_{2}}-1> \\
\phi\left(\left(a_{i, j}\right)\right) \longmapsto \sum_{i=0}^{n_{1}-1} \sum_{j=0}^{n_{2}-1} a_{i, j} x^{i} y^{j} .
\end{gathered}
$$

Proposition 1.3.2 A linear code $C \subseteq \mathbb{F}_{q}^{n_{1} \times n_{2}}$ is 2-D cyclic if and only if $\phi(C)$ is an ideal of $\mathbb{F}_{q}[x, y] /<x^{n_{1}}-1, y^{n_{2}}-1>$.

Proof: Being closed under row and column shifts are equivalent to being closed under multiplication by $x$ and $y$, respectively. Rest of the proof is clear.

From now on we assume that $n_{1}$ and $n_{2}$ are relatively prime to $p=\operatorname{char}\left(\mathbb{F}_{q}\right)$. Let $\alpha_{1}$ be a primitive $n_{1}$ th root of unity and $\alpha_{2}$ be a primitive $n_{2}$ th root of unity. We take both of these elements in the smallest extension $\mathbb{F}_{q^{s}}$ of $\mathbb{F}_{q}$ such that $n_{1}$ and $n_{2}$ divide $q^{s}-1$. Consider the following set:

$$
\Omega=\left\{\left(\alpha_{1}^{i}, \alpha_{2}{ }^{j}\right) \mid 0 \leq i \leq n_{1}-1,0 \leq j \leq n_{2}-1\right\} .
$$

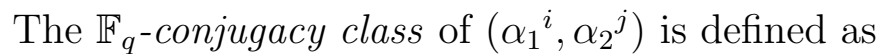

$$
\left[\left(\alpha_{1}{ }^{i}, \alpha_{2}{ }^{j}\right)\right]=\left\{\left(\alpha_{1}{ }^{i}, \alpha_{2}{ }^{j}\right),\left(\alpha_{1}{ }^{i q}, \alpha_{2}{ }^{j q}\right),\left(\alpha_{1}{ }^{i q^{2}}, \alpha_{2}{ }^{j q^{2}}\right), \cdots,\left(\alpha_{1}{ }^{i q^{\delta-1}}, \alpha_{2}{ }^{j q^{\delta-1}}\right)\right\},
$$

where $\delta$ is the least common multiple of the degrees of $\alpha_{1}{ }^{i}$ and $\alpha_{2}{ }^{j}$ over $\mathbb{F}_{q}$. We can write $\Omega$ as a disjoint union of these $\mathbb{F}_{q}$-conjugacy classes [4].

From now on, $U \subseteq \Omega$ will be the union of some conjugacy classes. The ideal corresponding to the set $U$ is defined as $I(U)=\left\{f(x, y) \in \mathbb{F}_{q}[x, y] \mid f(a)=0\right.$ for any $a \in U\}$. 
Remark 1.3.2 Note that for any $U \subseteq \Omega$, both of $x^{n_{1}}-1$ and $y^{n_{2}}-1$ are in $I(U)$. Therefore, $\left\langle x^{n_{1}}-1, y^{n_{2}}-1>\subseteq I(U)\right.$ and we can consider $\tilde{I}(U)=I(U) /<x^{n_{1}}-1, y^{n_{2}}-1>$. The ideal $\tilde{I}(U)$ is called the 2 -D cyclic code associated to $U \subseteq \Omega$.

Definition 1.3.3 Let $\tilde{J}=J /<x^{n_{1}}-1, y^{n_{2}}-1>$ be a 2 -D cyclic code. Then the set

$$
Z(\tilde{J})=\{(\alpha, \beta) \in \Omega \mid f(\alpha, \beta)=0 \text { for any } f \in J\}
$$

is called the zero set of the 2 -D cyclic code $\tilde{J}$.

Example 1.3.3 Let $q=2, n_{1}=3$, and $n_{2}=5$. We fix a primitive root of unity $\alpha \in \mathbb{F}_{16}$ which satisfies the equation $x^{4}+x+1=0$. So $\alpha^{5}$ and $\alpha^{3}$ are 3 rd and 5 th roots of unity, respectively. Put $\alpha_{1}=\alpha^{5}$ and $\alpha_{2}=\alpha^{3}$. Then $\Omega$ is

$$
\Omega=\left\{\left(\alpha_{1}^{i}, \alpha_{2}^{j}\right) \mid 0 \leq i \leq 2,0 \leq j \leq 4\right\} .
$$

Let $C$ be a 2-D cyclic code with the polynomial representation

$$
\tilde{I}=<(x+1)\left(y^{4}+y^{3}+y^{2}+y+1\right),(y+1)\left(x^{2}+x+1\right)>/<x^{3}-1, y^{5}-1>.
$$

Then

$$
Z(C)=[(1,1)] \cup\left[\left(\alpha_{1}, \alpha_{2}\right)\right] \cup\left[\left(\alpha_{1}^{2}, \alpha_{2}\right)\right]
$$

Now, we present the following statements without proof. See [4,9] for further details and proofs.

Proposition 1.3.4 Let $U \subseteq \Omega$. Then $Z(\tilde{I}(U))=U$

Theorem 1.3.5 Let $U$ be a subset of $\Omega$ and $\bar{U}=\Omega-U$. Consider the $2-D$ cyclic code $C_{U}$ corresponding to the ideal $I(U) /<x^{n_{1}}-1, y^{n_{2}}-1>$. Then dimension of the code $C_{U}$ is equal to $|\bar{U}|$.

Proposition 1.3.6 Let $C_{U}$ be the 2-D cyclic code with the zero set $U$ and polynomial representation $I(U) /<x^{n_{1}}-1, y^{n_{2}}-1>$. Then its dual is the 2 -D cyclic code $C_{\bar{U}^{-1}}$, which has the zero set

$$
Z\left(C_{U}^{\perp}\right)=Z\left(C_{\bar{U}^{-1}}\right)=\bar{U}^{-1}=\Omega-U^{-1},
$$

where $U^{-1}=\left\{\left(\mu_{1}^{-1}, \mu_{2}^{-1}\right) \mid\left(\mu_{1}, \mu_{2}\right) \in U\right\}$.

\subsection{Hermitian Dual of 2-D Cyclic Codes}

In this section, we assume that $\mathbb{F}_{q}$ is a finite field, where $q=p^{2}$ and $p$ is a prime number. Denote the conjugate of $a \in \mathbb{F}_{q}$ by $\bar{a}$, where $\bar{a}=a^{p}$. For $a, b \in \mathbb{F}_{q}^{n}$ we denote the Hermitian inner product of $a$ and $b$ with $\langle a, b\rangle=\sum_{i=1}^{n} a_{i} \overline{b_{i}}$. If $f(x, y)=\sum_{i=0}^{s} \sum_{j=0}^{t} a_{i, j} x^{i} y^{j}$ is a polynomial in $\mathbb{F}_{q}[x, y]$ we define $\overline{f(x, y)}=\sum_{i=0}^{s} \sum_{j=0}^{t} \overline{a_{i, j}} x^{i} y^{j}$. 
Lemma 1.4.7 Let $f(x, y)$ and $g(x, y)$ be polynomials in $\mathbb{F}_{q}[x, y]$. Then the following hold:

1. $\overline{f(x, y)+g(x, y)}=\overline{f(x, y)}+\overline{g(x, y)}$.

2. $\overline{f(x, y) \cdot g(x, y)}=\overline{f(x, y)} \cdot \overline{g(x, y)}$.

3. $\overline{\overline{f(x, y)}}=f(x, y)$

Corollary 1.4.8 For any ideal $I$ in $F_{q}[x, y], \bar{I}=\{\bar{f} \mid f \in I\}$ is also an ideal of $F_{q}[x, y]$.

It is clear that $\overline{x^{n_{1}}-1}=x^{n_{1}}-1$ and $\overline{y^{n_{2}}-1}=y^{n_{2}}-1$. So, if $\tilde{I}(U)$ is an ideal of $\mathbb{F}_{q}[x, y] /<x^{n_{1}}-1, y^{n_{2}}-1>$, then $\bar{I}(U)$ is also an ideal of $\mathbb{F}_{q}[x, y] /<x^{n_{1}}-1, y^{n_{2}}-1>$.

Lemma 1.4.9 Let $\tilde{I}(U)$ be an ideal of $\mathbb{F}_{q}[x, y] /<x^{n_{1}}-1, y^{n_{2}}-1>$. Then $\tilde{I}(U)$ and $\overline{\tilde{I}(U)}$ are isomorphic as ideals, hence as linear codes.

Proof: Consider the map

$$
\begin{gathered}
\phi: I(U) /<x^{n_{1}}-1, y^{n_{2}}-1>\longrightarrow \overline{I(U)} /<x^{n_{1}}-1, y^{n_{2}}-1> \\
f(x, y)+<x^{n_{1}}-1, y^{n_{2}}-1>\longmapsto \overline{f(x, y)}+<x^{n_{1}}-1, y^{n_{2}}-1>.
\end{gathered}
$$

Assume that $f(x, y)$ and $g(x, y) \in \mathbb{F}_{q}[x, y]$ and $f(x, y)+<x^{n_{1}}-1, y^{n_{2}}-1>=$ $g(x, y)+<x^{n_{1}}-1, y^{n_{2}}-1>$. Then $f(x, y)-g(x, y) \in<x^{n_{1}}-1, y^{n_{2}}-1>$ and consequently we can consider it as $f(x, y)-g(x, y)=h(x, y)\left(x^{n_{1}}-1\right)+k(x, y)\left(y^{n_{2}}-1\right)$ for some $h(x, y)$ and $k(x, y)$ in $\mathbb{F}_{q}[x, y]$. Hence $\overline{f(x, y)}-\overline{g(x, y)}=\overline{h(x, y)}\left(x^{n_{1}}-1\right)+$ $\overline{k(x, y)}\left(y^{n_{2}}-1\right)$ which means that $\overline{f(x, y)}+<x^{n_{1}}-1, y^{n_{2}}-1>=\overline{g(x, y)}+<x^{n_{1}}-$ $1, y^{n_{2}}-1>$. This shows that the map $\phi$ is well defined. By similar steps we can also show that $\phi$ is one-to-one. Moreover, according to Lemma 1.4.7, $\phi$ is a ring homomorphism. Finally, $\phi$ is onto since the conjugation operator is an automorphism of $\mathbb{F}_{q}$.

Theorem 1.4.10 Let $\tilde{I}(U)$ be an ideal of $\mathbb{F}_{q}[x, y] /<x^{n_{1}}-1, y^{n_{2}}-1>$. Then the zero set of the ideal $\overline{\tilde{I}}(U)$ is the set $U^{p}=\left\{\left(\alpha^{p}, \beta^{p}\right) \mid(\alpha, \beta) \in U\right\}$.

Proof: By Lemma 1.4.9, $\operatorname{dim}(\tilde{I}(U))=\operatorname{dim}(\overline{\tilde{I}}(U))$. Also, since $p$ is relatively prime to both $n_{1}$ and $n_{2},\left|U^{p}\right|=|U|$. So it is enough to show that $U^{p} \subseteq Z(\overline{\tilde{I}}(U))$. Let $\overline{f(x, y)} \in \overline{\tilde{I}(U)}$, where $f(x, y) \in \tilde{I}(U)$. We have

$$
\overline{f(x, y)}=\sum_{i=0}^{n_{1}-1} \sum_{j=0}^{n_{2}-1} \overline{a_{i, j}} x^{i} y^{j}=\sum_{i=0}^{n_{1}-1} \sum_{j=0}^{n_{2}-1} a_{i, j}{ }^{p} x^{i} y^{j} .
$$

Now, suppose $\left(\alpha^{p}, \beta^{p}\right) \in U^{p}$. Then,

$$
\overline{f\left(\alpha^{p}, \beta^{p}\right)}=\sum_{i=0}^{n_{1}-1} \sum_{j=0}^{n_{2}-1} a_{i j}^{p} \alpha^{i p} \beta^{j p}=\left(\sum_{i=0}^{n_{1}-1} \sum_{j=0}^{n_{2}-1} a_{i j} \alpha^{i} \beta^{j}\right)^{p}=(f(\alpha, \beta))^{p}=0 .
$$

Therefore, $U^{p} \subseteq Z(\overline{\tilde{I}}(U))$.

Now, we present the main theorem of this section which allows us to find the Hermitian dual of an arbitrary 2-D cyclic code. 
Theorem 1.4.11 Let $C_{U}$ be the 2-D cyclic code with the zero set $U$. Then, the Hermitian dual of $C_{U}$ is the code $C_{\bar{U}^{-p}}$, which has the zero set $\bar{U}^{-p}=\Omega-U^{-p}$.

Proof: Let $I(U) /<x^{n_{1}}-1, y^{n_{2}}-1>$ be the polynomial representation of the 2-D cyclic code $C_{U}$. By Proposition 1.3.6, the Euclidean dual of $C_{U}$ is the code $C_{\bar{U}^{-1}}$, which has the polynomial representation $I\left(\bar{U}^{-1}\right) /<x^{n_{1}}-1, y^{n_{2}}-1>$. Now, let $\overline{C_{\bar{U}^{-1}}}$ be the code corresponding to the conjugate of the ideal $I\left(\bar{U}^{-1}\right) /<x^{n_{1}}-1, y^{n_{2}}-1>$. By Theorem 1.4.10, we conclude that $\overline{C_{\bar{U}^{-1}}}=C_{\bar{U}^{-p}}$.

Now, we claim that the Hermitian dual of the code $C_{U}$ is $\overline{C_{\bar{U}^{-1}}}$. It's because of that

$$
I(U) \cdot \overline{I\left(\bar{U}^{-p}\right)} \equiv I(U) \cdot I\left(\bar{U}^{-1}\right) \quad \bmod <x^{n_{1}}-1, y^{n_{2}}-1>
$$

and since $I\left(\bar{U}^{-1}\right) /<x^{n_{1}}-1, y^{n_{2}}-1>$ is the Euclidean dual of $I(U) /<x^{n_{1}}-1, y^{n_{2}}-1>$, we have:

$$
I(U) \cdot I\left(\bar{U}^{-1}\right) \equiv 0 \quad \bmod <x^{n_{1}}-1, y^{n_{2}}-1>.
$$

Note that in the proof of the last theorem, the fact $\left(\bar{U}^{-p}\right)^{p}=\bar{U}^{-1}$ is used which can be easily verified.

Example 1.4.12 Let $q=4$ and $n_{1}=n_{2}=3$. We fix a primitive root of unity $\alpha \in \mathbb{F}_{4}=\{0,1, \alpha, \bar{\alpha}\}$ which satisfies the equation $x^{2}+x+1=0$. So we can put $\alpha_{1}=\alpha_{2}=\alpha$. Then $\Omega$ is

$$
\Omega=\left\{\left(\alpha_{1}^{i}, \alpha_{2}{ }^{j}\right) \mid 0 \leq i \leq 2,0 \leq j \leq 2\right\} .
$$

Let $C$ be the 2 -D cyclic code over $\mathbb{F}_{4}$ with the polynomial representation

$$
\tilde{I}=<(x+1)\left(y^{2}+\alpha y+1\right),(y+1)\left(x^{2}+x+1\right)>/<x^{n_{1}}-1, y^{n_{2}}-1>.
$$

Then

$$
Z(C)=\left\{(1,1),\left(\alpha_{1}, \alpha_{2}^{2}\right),\left(\alpha_{1}, 1\right),\left(\alpha_{1}^{2}, 1\right),\left(\alpha_{1}^{2}, \alpha_{2}^{2}\right)\right\} .
$$

Note that all of these elements have a singleton conjugacy class. Now, according to Theorem 1.4.11, the zero set of the Hermitian dual of $C$ is

$$
Z\left(C^{\perp_{h}}\right)=\Omega-(Z(C))^{-2}=\left\{\left(1, \alpha_{2}\right),\left(1, \alpha_{2}^{2}\right),\left(\alpha_{1}, \alpha_{2}\right),\left(\alpha_{1}^{2}, \alpha_{2}\right)\right\} .
$$

Also, this set is the zero set of the ideal

$$
<(y+\alpha)(x+1),\left(x^{2}+x+1\right)\left(y^{2}+y+1\right)>/<x^{n_{1}}-1, y^{n_{2}}-1>.
$$

Therefore, $C^{\perp_{h}}=<(y+\alpha)(x+1),\left(x^{2}+x+1\right)\left(y^{2}+y+1\right)>/<x^{n_{1}}-1, y^{n_{2}}-1>$.

Now, we state another theorem which plays a critical role in finding quantum stabilizer codes in Chapter 3.

Theorem 1.4.13 If $C_{U}$ is the 2-D cyclic code with the zero set $U$, then $\operatorname{dim}\left(C_{U}{ }^{\perp_{h}}\right)-$ $\operatorname{dim}\left(C_{U} \cap C_{U}^{\perp_{h}}\right)=\left|U \cap U^{-p}\right|$. 
Proof: By Theorem 1.3.5, $\operatorname{dim}\left(C_{U}\right)=|\bar{U}|=|\Omega-U|$. Also, from Theorem 1.4.11, we can see that $Z\left(C_{U}^{\perp_{h}}\right)=\bar{U}^{-p}$. It is clear that $Z\left(C_{U} \cap C_{U}^{\perp_{h}}\right)=U \cup \bar{U}^{-p}$. So,

$$
\begin{aligned}
\operatorname{dim}\left(C_{U}^{\perp_{h}}\right)-\operatorname{dim}\left(C_{U} \cap C_{U}^{\perp_{h}}\right) & =\left|\Omega-\bar{U}^{-p}\right|-\left|\Omega-\left(U \cup \bar{U}^{-p}\right)\right| \\
& =n_{1} n_{2}-\left|\bar{U}^{-p}\right|-\left(n_{1} n_{2}-\left|U \cup \bar{U}^{-p}\right|\right) \\
& =\left|U \cup \bar{U}^{-p}\right|-\left|\bar{U}^{-p}\right| \\
& =\left|U-\bar{U}^{-p}\right|=\left|U \cap U^{-p}\right| .
\end{aligned}
$$

The last equality follows from the fact that $U=\left(U \cap U^{-p}\right) \dot{\cup}\left(U \cap \bar{U}^{-p}\right)$.

\subsection{Characterization of 2-D Cyclic Codes}

In this Section, we give a characterization of 2-D cyclic codes using the polynomial representation of the codes [11]. For this purpose, we assume that $I$ is an ideal of the ring $\mathbb{F}_{q}[x, y] /<x^{n_{1}}-1, y^{n_{2}}-1>$ and $f(x, y) \in I$. Then $f(x, y)$ can be written as $f(x, y)=\sum_{i=0}^{n_{2}-1} f_{i}(x) y^{i}$, where each $f_{i}(x)$ belongs to $R=\mathbb{F}_{q}[x] /<x^{n_{1}}-1>$. We define the set

$$
I_{0}=\left\{g_{0}(x) \in R \mid \text { there exists } g(x, y) \in I \text { such that } g(x, y)=\sum_{i=0}^{n_{2}-1} g_{i}(x) y^{i}\right\} .
$$

Clearly $I_{0}$ is an ideal of the $\operatorname{ring} R$. Since $R$ is a principal ideal ring, we can write $I_{0}=<p_{0}^{0}(x)>$, where $p_{0}^{0}(x) \in R$ and $p_{0}^{0}(x) \mid x^{n_{1}}-1$. So for each $f(x, y) \in I$, we have $f_{0}(x) \in I_{0}$ and therefore $p_{0}^{0}(x) \mid f_{0}(x)$. On the other hand, $p_{0}^{0}(x) \in I_{0}$ which implies that there exists $p_{0}(x, y)=\sum_{i=0}^{n_{2}-1} p_{i}^{0}(x) y^{i} \in I$. Hence, for every $f(x, y) \in I$ we can find $q_{0}(x) \in R$ such that $h(x, y)=f(x, y)-q_{0}(x) p_{0}(x, y)$ belongs to $I$ and has no constant term relative to $y$. This means that if $h(x, y)=\sum_{i=0}^{n_{2}-1} h_{i}(x) y^{i}$, then $h_{0}(x)=0$. Now, put

$$
I_{1}=\left\{g_{1}(x) \in R \mid \text { there exists } g(x, y) \in I \text { such that } g(x, y)=\sum_{i=1}^{n_{2}-1} g_{i}(x) y^{i}\right\} .
$$

Again, $I_{1}$ is an ideal of $R$ which is generated by $p_{1}^{1}(x)$, where $p_{1}^{1}(x) \in R$ and $p_{1}^{1}(x) \mid x^{n_{1}}-1$. Moreover, $p_{1}^{1}(x) \in I_{1}$ which means that there exists $p_{1}(x, y) \in I$ such that $p_{1}(x, y)=$ $\sum_{i=1}^{n_{2}-1} p_{i}^{1}(x) y^{i}$. Now, for each $f(x, y) \in I$ we can find $q_{0}(x)$ and $q_{1}(x) \in R$ such that $h^{\prime}(x, y)=f(x, y)-q_{0} p_{0}(x, y)-q_{1} p_{1}(x . y)$ has no constant and degree one term relative to $y$. In other words, if $h^{\prime}(x, y)=\sum_{i=0}^{n_{2}-1} h_{i}^{\prime}(x) y^{i}$, then $h_{0}^{\prime}=h_{1}^{\prime}=0$. Repeating this idea, we can construct $p_{i}(x, y) \in I$ for $i=0,1,2, \cdots, n_{2}-1$. Now, it is clear that

$$
I=<p_{0}(x, y), p_{1}(x, y), \cdots, p_{n_{2}-1}(x, y)>.
$$


The polynomials $p_{i}(x, y)$ are called the generator polynomials of $I$. By the above construction, we can find some conditions on $p_{i}(x, y)$.

Proposition 1.5.14 Let $p_{i}(x, y) \in I$ for $i=0,1,2, \cdots, n_{2}-1$ be the polynomials obtained from the above construction. Then we have the followinng properties.

(i) $p_{0}^{0}(x) \mid p_{i}^{j}(x)$ for all $0 \leq i, j \leq n_{2}-1$.

(ii) $p_{i-1}^{i-1}(x) \mid p_{i}^{i}(x)$.

(iii) $p_{i}^{i}(x) \mid \frac{x^{n_{1}-1}}{p_{i-1}^{i-1}}(x) p_{i}^{i-1}(x)$.

Proof: $(i)$ By the definition of $I_{0}$, we have $p_{i}^{j}(x) \in I_{0}$ for all $0 \leq i, j \leq n_{2}-1$. Since $p_{0}^{0}(x)$ is the generator polynomial of the ideal $I_{0}$, we have $p_{0}^{0}(x) \mid p_{i}^{j}(x)$.

(ii) For $i \leq j$, we have $I_{j} \subseteq I_{i}$, hence $p_{i-1}^{i-1}(x) \mid p_{i}^{i}(x)$.

(iii) Let $P_{i-1}(x, y)=\sum_{j=i-1}^{n_{2}-1} p_{j}^{i-1}(x) y^{j}$. Consider the polynomial

$$
s(x, y)=\frac{x^{n_{1}-1}}{p_{i-1}^{i-1}(x)} P_{i-1}(x, y)=\sum_{j=i}^{n_{2}-1} p_{j}^{i-1}(x) \frac{x^{n_{1}}-1}{p_{i-1}^{i-1}(x)} y^{j} .
$$

We have, $s_{i}=\frac{x^{n_{1}}-1}{p_{i-1}^{i-1}(x)} p_{i-1}^{i}(x) \in I_{i}$, so $p_{i}^{i}(x) \mid \frac{x^{n_{1}}-1}{p_{i-1}^{i-1}}(x) p_{i}^{i-1}(x)$.

Theorem 1.5.15 Let $I$ be an ideal of the ring $\mathbb{F}_{q}[x, y] /<x^{n_{1}}-1, y^{n_{2}}-1>(a 2-D$ cyclic code). Then we have

$$
I=<p_{0}(x, y), p_{1}(x, y), \cdots, p_{n_{2}-1}(x, y)>,
$$

where $p_{i}(x, y)$ 's are defined as above. Moreover, the set

$$
S=\bigcup_{i=0}^{n_{2}-1}\left\{P_{i}(x, y), x P_{i}(x, y), x^{2} P_{i}(x, y), \cdots, x^{n_{1}-a_{i}-1} P_{i}(x, y)\right\}
$$

where $a_{i}=\operatorname{deg}\left(p_{i}^{i}(x)\right)$ for $0 \leq i \leq n_{2}-1$, is an $\mathbb{F}_{q}$-basis for the 2 -D cyclic code.

Proof: Elements of $S$ generate $I$. It is enough to show that they are linearly independent. Assume that they are linearly dependent. So there are $k_{0}(x), k_{1}(x), \cdots, k_{n_{2}-1}(x) \in$ $\mathbb{F}_{q}[x] /<x^{n_{1}}-1>$ such that $\operatorname{deg}\left(k_{i}(x)\right) \leq n_{1}-a_{i}-1$ and $\sum_{i=0}^{n_{2}-1} k_{i}(x) p_{i}(x, y)=0$ in $\mathbb{F}_{q}[x] /<x^{n_{1}}-1>$. This means that $k_{0}(x) p_{0}^{0}(x)=0$ in $\mathbb{F}_{q}[x] /<x^{n_{1}}-1>$. So, $k_{0}(x) p_{0}^{0}(x)=t(x)\left(x^{n_{1}}-1\right)$ for some $t(x) \in \mathbb{F}_{q}[x]$, but $\operatorname{deg}\left(k_{0}(x) p_{0}^{0}(x)\right)<n_{1}-1$ and it implies that $k_{0}(x)=0$. Similarly we can show that $k_{i}(x)=0$ for $0 \leq i \leq n_{2}-1$ and this completes the proof. 


\section{CHAPTER 2}

\section{Quantum Error Correction Codes}

\subsection{Binary Stabilizer Quantum Codes}

Let $\mathbb{F}_{4}$ be the finite field of the elements $\left\{0,1, w, w^{2}\right\}$, with $w^{2}=w+1$. We define the trace map $\operatorname{Tr}: \mathbb{F}_{4} \rightarrow \mathbb{F}_{2}$ with $\operatorname{Tr}(x)=x+\bar{x}$ where $\bar{x}=x^{2}$. An additive subgroup of $\mathbb{F}_{4}^{n}$ is called an additive code. Let $C \subseteq \mathbb{F}_{4}^{n}$ be an additive code over $\mathbb{F}_{4}$, then the Hamming weight of $v \in C$ is the number of nonzero components of $v$. Consequently, the distance of two vectors $u, v \in C$ is $\operatorname{dist}(u, v)=w t(u-v)$. Now, we can define the minimum distance of the code $C$ with $\min \{\operatorname{dist}(u, v) \mid u \neq v \in C\}$.

We define the symplectic inner product of $u, v \in C$ with

$$
u * v=\operatorname{Tr}(u \cdot \bar{v})=(u \cdot \bar{v})+\overline{(u \cdot \bar{v})}=\sum_{i=1}^{n}\left(u_{i} \overline{v_{i}}+\overline{u_{i}} v_{i}\right) .
$$

If $C$ is an $\left(n, 2^{k}\right)$ code its symplectic dual will be $C^{\perp_{s}}=\left\{u \in \mathbb{F}_{4}^{n} \mid u * v=0\right.$ for all $v \in C\}$. It is clear that $C^{\perp_{s}}$ is an $\left(n, 2^{2 n-k}\right)$ additive code. We say $C$ is self-orthogonal if $C \subseteq C^{\perp_{s}}$. Also, if $C=C^{\perp_{s}}$ we call the code $C$ a self-dual code.

Lemma 2.1.1 Suppose $C \subseteq \mathbb{F}_{4}^{n}$ is an $\left(n, 2^{n-k}\right)$ additive self-orthogonal code such that $d=\min \left\{w t(u) \mid u \in C^{\perp_{s}} \backslash C\right\}$. Then $C$ can be used to construct a binary $[[n, k, d]]$ quantum stabilizer code. [3]

Definition 2.1.1 From now on, for simplicity, if the block code $C$ satisfies the conditions of Lemma 2.1.1, we call $C$ as $[[n, k, d]]$ quantum (stabilizer) code. If the block code $C$ is linear, wel call the code linear quantum code.

We call $C$ pure if there is no nonzero vector in $C^{\perp_{s}}$ with weight less than $d$, otherwise it called impure. Note that the quantum code $C$ with parameters $[[n, 0, d]]$ is a pure quantum code. In this special case, $k=0$, we define $d=\min \{w t(u) \mid u \in C \backslash\{0\}\}$. In the next theorem, we show that Hermitian dual and symplectic dual are the same if $C$ is an $\mathbb{F}_{4}$ linear code.

Theorem 2.1.2 An $\mathbb{F}_{4}$ linear code $C$ is self-orthogonal with respect to the symplectic inner product if and only if it is self-orthogonal with respect to the Hermitian inner product. 
Proof: Suppose that $C$ is self-orthogonal linear code with respect to the symplectic inner product. Then we have $u * v=\operatorname{Tr}(u \cdot \bar{v})=0$ for all $u, v \in C$. If $u \cdot \bar{v}=\alpha+\beta w$ with $\alpha, \beta \in \mathbb{F}_{2}$, then $\bar{u} \cdot v=\overline{u \cdot \bar{v}}=\alpha+(w+1) \beta$. Therefore, $u * v=u \cdot \bar{v}+\bar{u} \cdot v=\beta$ which means that $\beta=0$. Moreover, since $C$ is $\mathbb{F}_{4}$ linear, $w v \in C$ and $u * w v=\operatorname{Tr}(u \cdot \overline{w v})=0$. Using the same argument we see that $\alpha=0$ and consequently $u \cdot \bar{v}=0$ for all $u$ and $v \in C$. Proof of the other direction is straightforward.

An $\left(n, 2^{k}\right)$ additive code over $\mathbb{F}_{4}$ is called even if the weight of each codeword in $C$ is even. Otherwise, $C$ is called odd.

Theorem 2.1.3 An even additive code is self-orthogonal. The converse is correct for linear codes.

Proof: Let $C$ be an additive even code and $u, v \in C$. Let $S_{1}$ and $S_{2}$ be subsets of coordinates such that $u$ and $v$ have the same entries at each place of $S_{1}$ and different components of them occur in all the places of $S_{2}$. Then,

$$
w t(u+v)=w t(u)+w t(v)-2 k-r,
$$

where $k$ is the number of coordinates of $S_{1}$ such that $u$ and $v$ are nonzero in those places, and $r$ is the number of places of $S_{2}$ such that $u$ and $v$ are both nonzero and different. By definition of the symplectic inner product, we see that $r=u * v$. So

$$
w t(u+v)=w t(u)+w t(v)-2 k-r \equiv w t(u)+w t(v)+u * v(\bmod 2) .
$$

Since the code is even, $u * v=0$. Conversely, if $C$ is a linear code, then $u w \in C$ for all $u \in C$. Also,

$$
0 \equiv u * w u=w t(u)(\bmod 2)
$$

and this completes the proof.

\subsection{General Constructions of Quantum Stabilizer Codes}

In this Section, we will introduce some general methods of constructing and modifying quantum codes over $\mathbb{F}_{4}$. We define the direct sum of two quantum codes as $C \oplus C^{\prime}=\left\{u v \mid u \in C, v \in C^{\prime}\right\}$. Clearly, if $C$ and $C^{\prime}$ are $[[n, k, d]]$ and $\left[\left[n^{\prime}, k^{\prime}, d^{\prime}\right]\right]$ quantum codes respectively, then $C \oplus C$ is an $\left[\left[n+n^{\prime}, k+k^{\prime}, d^{\prime \prime}\right]\right]$ quantum code, where $d^{\prime \prime}=\min \left\{d, d^{\prime}\right\}$. Additive codes which cannot be expressed as a direct sum are called indecomposable. The next theorem introduces other construction methods for quantum codes.

Theorem 2.2.4 Suppose $C$ is an $[[n, k, d]]$ quantum code.

(i) If $k>0$, then there exists a quantum code $C^{\prime}$ with parameters $[[n+1, k, d]]$.

(ii) If $C$ is pure and $n \geq 2$, then an $[[n-1, k+1, d-1]]$ quantum code exists.

(iii) If $k>1$ or if $k=1$ and $C$ is pure, then an $[[n, k-1, d]]$ quantum code exists.

(iv) If $n \geq 2$, then an $[[n-1, k, d-1]]$ quantum code exists.

(v) If $n \geq 2$ and $C$ contains a codeword of weight 1 , then an $[[n-1, k, d]]$ quantum code exists. 
Proof: $(i)$ Let $C$ be an $\left(n, 2^{n-k}\right)$ quantum code, where $k>0$. Consider $C^{\prime}=C \oplus C_{1}$, where $C_{1}=\{0,1\}$. Obviously, $C^{\prime}$ is self-orthogonal with respect to the symplectic inner product, it has $2^{n-k+1}$ elements, and its dual can be written as $C^{\prime \perp}=C^{\perp} \oplus C_{1}$. Therefore, it is an $[[n+1, k, d]]$ quantum code. Not that this construction dose not hold when $k=0$. Since otherwise we can construct an $[[n+1,0]]$ code. Moreover, since $0 \oplus 1 \in C^{\prime}$ and the code is pure, the minimum distance is 1 .

(ii) Since $C$ is a pure code, by deleting the first coordinate of the code $C^{\perp}$ we obtain $B^{\perp}$ with parameters $\left(n-1,2^{n+k}\right)$ and minimum distance at least $d-1$. So, it is enough to show that $B \subseteq B^{\perp}$. We claim that $B=\{v \mid 0 v \in C\}$. By definition of symplectic inner product $\{v \mid 0 v \in C\} \subseteq B$. Also, if $u \in B$ then $0 u * c=0$ for all $c \in C^{\perp}$ and this means that $B \subseteq B^{\perp}$.

(iii) First, suppose that $k>1$ and $v \in C^{\perp} \backslash C$. Then the code $C^{\prime}$ generated by $C^{\prime}=\left\langle C, v>\right.$ has parameters $\left(n, 2^{n-k+1}\right)$ and it is self-orthogonal with respect to the symplectic inner product. Since $C^{\perp} \backslash C^{\prime} \subseteq C^{\perp} \backslash C$, it has minimum distance $\geq d$. So, $C^{\prime}$ is an $[[n, k-1, d]]$ quantum code. Now, in case $k=1$, since the construction ends in an $[[n, 0]]$ quantum code, which is a pure code and we cannot grantee the minimum distance $d$ (in pure code we calculate minimum distance differently).

(iv) Take $B=\{u \mid 0 u$ or $1 u \in C\}$. We show that $B^{\perp}=\left\{v \mid 0 v\right.$ or $\left.1 v \in C^{\perp}\right\}$.It is clear that if $0 v$ or $1 v \in C^{\perp}$, then for any $u \in B$ we have $v * u=0$. So $\left\{v \mid 0 v\right.$ or $\left.1 v \in C^{\perp}\right\} \subseteq B^{\perp}$. Conversely, suppose that $v \in B^{\perp}$ and, both of $0 v$ and $1 v$ are not in $C^{\perp}$. First, since $0 v \notin C^{\perp}$, then there exist $s=\left(s_{1}, s_{2}, \cdots, s_{n}\right) \in C$ such that $s_{1} \in\{w, w+1\}$ and $0 v * s \equiv 1 \bmod 2$. So $\left(s_{2}, s_{3} . \cdots, s_{n}\right) * v \equiv 1 \bmod 2$. Next, since $1 v \notin C^{\perp}$, there exists $r=\left(r_{1}, r_{2}, \cdots, r_{n}\right) \in C$ such that $r_{1} \in\{w, w+1\}$ and $1 v * r \equiv 1 \bmod 2$. Therefore, $\left(r_{2}, r_{3}, \cdots, r_{n}\right) * v \equiv 0 \bmod 2$. Now, $s+r=\left(z_{1}, z_{2}, \cdots, z_{n}\right) \in C$, where $z_{1} \in\{0,1\}$. Hence, $\left(z_{2}, z_{3}, \cdots, z_{n}\right) \in B$, which means that $v *\left(z_{2}, z_{3}, \cdots, z_{n}\right) \equiv 0 \bmod 2$. But, $v *\left(z_{2}, z_{3}, \cdots, z_{n}\right)=v *\left(s_{2}, s_{3} . \cdots, s_{n}\right)+v *\left(r_{2}, r_{3} \cdots, r_{n}\right) \equiv 1+0 \equiv 1 \bmod 2$, and this is a contradiction. So, $B^{\perp}=\left\{v \mid 0 v\right.$ or $\left.1 v \in C^{\perp}\right\}$.

Now, if there is a vector with first coordinate $w$ or $w+1$ in $C$, then $|B| \leq 2^{n-k-1}$. Otherwise, if $0 u(1 v)$ is a vector in $C$, then $1 u(0 v)$ is also a vector of $C^{\perp}$ and again we conclude that $|B| \leq 2^{n-k-1}$. Moreover, since $C \subseteq C^{\perp}$ we can see that $\left|B^{\perp}\right| \leq 2^{n+k-1}$. On the other hand, $\operatorname{dim}(B)+\operatorname{dim}\left(B^{\perp}\right)=2 n-2$. Hence, $|B|=2^{n-k-1}$. Now we consider the minimum distance. If $t \in C^{\perp} \backslash C, t$ starts with 1 , and has weight $d$, then $t$ after truncation is a codeword in $B^{\perp} \backslash B$ and has weight $\leq d-1$. This is why we the minimum distance of new code is $d-1$.

$(v)$ Suppose $v \in C$ such that for some $1 \leq i \leq n, v_{i}=a \neq 0$ and $v_{j}=0$ for $i \neq j$. Note that for any $u \in C^{\perp}, u_{i}=0$ or $a$. So by deleting the $i$ th coordinate of $C$ and $C^{\perp}$, we obtain subspaces $B$ and $B^{\prime}$ such that $B^{\prime}=B^{\perp}$. This is because of the fact that if $s \in C$ and $t \in C^{\perp}$, the symplectic inner product of $i$ th coordinate is always equal to zero. Also, $\operatorname{dim}(B) \leq \operatorname{dim}(C)-1$ and $\operatorname{dim}\left(B^{\perp}\right) \leq \operatorname{dim}\left(C^{\perp}\right)-1$. On the other hand, $\operatorname{dim}(B)+\operatorname{dim}\left(B^{\perp}\right)=2 n-2$. So $B$ is an $[[n-1, k]]$ quantum code. Moreover, If $t \in C^{\perp} \backslash C$ with $w t(t)=d$, then $t+v \in C^{\perp} \backslash C$, so the $i$ th coordinate of $t$ is zero and this means $t \in B^{\perp} \backslash B$. This completes the proof.

Lemma 2.2.5 Let $C$ be a linear quantum code over $\mathbb{F}_{4}$. Suppose that $S$ is a subset of coordinates such that $S$ meets each vector of $C$ in an even weight vector. Then, the code obtained by deleting the coordinates $S$ is also a linear quantum code.

Proof: By Theorem 2.1.3, $C$ is an even code. Therefore, deleting coordinates corresponding to $S$, we obtain another even code, which is self-orthogonal by Theorem 
2.1.3. Hence, the resulting code is a quantum code again.

Theorem 2.2.6 Suppose $C$ is a linear quantum code with parameters $[[n, k, d]]$. Then there exists an $\left[\left[n-m, k^{\prime}, d^{\prime}\right]\right]$ linear quantum code, where $k^{\prime} \geq k-m$ and $d^{\prime} \geq d$, if there exists a codeword of weight $m$ in the dual of the binary code which is generated by the support coordinates of the code $C$.

Proof: Let $v$ be the mentioned codeword in the dual of the binary code generated by the supports of the code $C$. Assume that the set of nonzero coordinates of $v$ is equal to $S$. Then by Lemma 2.2.5, the code $C^{\prime}$ obtained by deleting the coordinates of $S$ is self -orthogonal and has more than $2^{n-k+m}$ vectors. Moreover, if there exists a codeword $u \in C^{\perp_{s}} \backslash C$ with $w t(u)=d^{\prime}$, then we replace the removed coordinate with zero in $u$ and clearly it is a codeword in $C$ with weight $\leq d^{\prime}$. Therefore, $d \leq d^{\prime}$.

Now, we present another result which generalizes the direct sum construction of two quantum stabilizer codes.

Theorem 2.2.7 Let $C_{1}$ and $C_{2}$ be two quantum stabilizer codes with the parameters $\left[\left[n_{1}, k_{1}, d_{1}\right]\right]$ and $\left[\left[n_{2}, k_{2}, d_{2}\right]\right]$, respectively. If $k_{2} \leq n_{1}$, we can construct an $\left[\left[n_{1}+n_{2}-\right.\right.$ $\left.\left.k_{2}, k_{1}, d\right]\right]$ quantum stabilizer code with $d \geq \min \left\{d_{1}, d_{1}+d_{2}-k_{2}\right\}$.

Proof: Let $C_{1}, C_{1}^{\perp_{s}}$ be additive codes with parameters $\left(n_{1}, 2^{n_{1}-k_{1}}\right),\left(n_{1}, 2^{n_{1}+k_{1}}\right)$ and $C_{2}, C_{2}^{\perp_{s}}$ be additive codes with the parameters $\left(n_{2}, 2^{n_{2}-k_{2}}\right),\left(n_{2}, 2^{n_{2}+k_{2}}\right)$, respectively. Let $N$ be the natural map from $C_{2}^{\perp_{s}}$ to $C_{2}^{\perp_{s}} / C_{2}$ and $F$ be a bijection from $C_{2}^{\perp} / C_{2}$ to $\mathbb{F}_{4}^{k_{2}}$ that preserves the inner product. Let $\phi=F \circ N$ be the composition of $F$ and $N$. Consider the code $C=\left\{u v \mid v \in C_{2}^{\perp}, u \phi(v) \in C_{1}\right\}$. Obviously, the length of $C$ is $n_{2}+n_{1}-k_{2} . \operatorname{dim}(C)=n_{2}+n_{1}-k_{2}-k_{1}$ because if $v \in C_{2}$, then $0 v \in C$ so we have $n_{2}-k_{2}$ linearly independent vectors. Also, since $\phi\left(C_{2}^{\perp} / C_{2}\right)=\mathbb{F}_{4}^{k_{2}}$ then we can find new $n_{1}-k_{1}$ linear independent vectors. We easily see that $C^{\perp}=\left\{u v \mid v \in C_{2}^{\perp}, u \phi(v) \in C_{1}{ }^{\perp}\right\}$. Now, if $u v \in C^{\perp}$ and $\phi(v) \neq 0, w t(v) \geq d_{2}$ and $w t(u) \geq d_{1}-k_{2}$. So $w t(u v) \geq d_{2}+d_{1}-k_{2}$. If $\phi(v)=0, w t(u) \geq d_{1}$ and consequently $w t(u v) \geq d_{1}$. This completes the proof.

For example, if $C_{1}$ and $C_{2}$ are $\left[\left[n_{1}, k_{1}, d_{1}\right]\right]$ and $[[1,0,1]]$ quantum stabilizer codes, respectively, then the code obtained by Theorem 2.2 .7 is an $\left[\left[n_{1}+1, k_{1}, d_{1}\right]\right]$ which is the same as the code obtained by Theorem 2.2.4 part $i$.

Now, we present another construction which is based on combining binary linear codes.

Theorem 2.2.8 Let $C_{1}$ be an $\left(n, 2^{k_{1}}\right), C_{2}$ be an $\left(n, 2^{k_{2}}\right)$ binary linear codes such that $C_{1} \subseteq C_{2}$, and $w \neq 0,1$ be an element of $\mathbb{F}_{4}$. Then the code $C=w C_{1}+\bar{w} C_{2}{ }^{\perp}$ is an $\left[\left[n, k_{2}-k_{1}, d\right]\right]$ quantum stabilizer code, where $d=\min \left\{d\left(C_{2} \backslash C_{1}\right), d\left(C_{1}{ }^{\perp} \backslash C_{2}{ }^{\perp}\right)\right\}$.

Proof: Since $C_{2}$ is a binary code, $\operatorname{dim}\left(C_{2}{ }^{\perp}\right)=n-k_{2}$. Therefore, $\operatorname{dim}(C)=k_{1}+n-k_{2}$. Let $w x+\bar{w} y$ and $w x^{\prime}+\bar{w} y^{\prime}$ be elements of $C$. Then

$$
\begin{aligned}
(w x+\bar{w} y) *\left(w x^{\prime}+\bar{w} y^{\prime}\right) & =w x * w x^{\prime}+w x * \bar{w} y^{\prime}+\bar{w} y * w x^{\prime}+\bar{w} y * \bar{w} y^{\prime} \\
& =w x * \bar{w} y^{\prime}+\bar{w} y * w x^{\prime}=x * y^{\prime}+x^{\prime} * y .
\end{aligned}
$$

Since $C_{1} \subseteq C_{2}$ we can conclude that $(w x+\bar{w} y) *\left(w x^{\prime}+\bar{w} y^{\prime}\right)=0$. Therefore, $C$ is an $\left[\left[n, k_{2}-k_{1}\right]\right]$ quantum code and $C^{\perp}=\bar{w} C_{1}{ }^{\perp}+w C_{2}$. Now, if $u \in C^{\perp} \backslash C$, then $u=\left(u_{1}, u_{2}\right)$, where $u_{1} \in w C_{2} \backslash w C_{1} \cup\{0\}$ and $u_{2} \in \bar{w} C_{1}{ }^{\perp} \backslash \bar{w} C_{2}{ }^{\perp} \cup\{0\}$. Thus, $d=$ $\min \left\{d\left(C_{2} \backslash C_{1}\right), d\left(C_{1}{ }^{\perp} \backslash C_{2}{ }^{\perp}\right)\right\}$.

In the next theorem, we explain an analog of the $(u \mid u+v)$ construction for quantum codes. This construction is also known as the Plotkin sum of two codes. 
Theorem 2.2.9 Suppose that $C_{1}$ is a pure $\left[\left[n, k_{1}, d_{1}\right]\right]$ stabilizer code, and $C_{2}$ is a pure $\left[\left[n, k_{2}, d_{2}\right]\right]$ stabilizer code such that $C_{1} \subseteq C_{2}$. Then the code $C=\{(u \mid u+v): u \in$ $\left.C_{2}{ }^{\perp}, v \in C_{1}\right\}$ is an $\left[\left[2 n, k_{1}-k_{2}, d\right]\right]$ stabilizer code, where $d=\min \left\{2 d_{1}, \delta\right\}, \delta=d\left(C_{2}\right)$.

Proof: Let $C_{1} \subseteq C_{2}$. Then, $\operatorname{dim}(C)=n+k_{2}+n-k_{1}$. Also, if $(u \mid u+v)$ and $(x \mid x+y) \in C$, then

$(u \mid u+v) *(x \mid x+y)=u * x+u * x+u * y+v * x+v * y=u * y+v * x+v * y=0$.

Thus, $C$ is an $\left[\left[2 n, k_{1}-k_{2}\right]\right]$ stabilizer code and $C^{\perp}=\left\{(u \mid u+v): u \in C_{1}^{\perp}, v \in C_{2}\right\}$. Assume that $(u \mid u+v) \in C^{\perp}$. If $u \neq 0, w t(u \mid u+v) \geq 2 d_{1}$ and if $u=0, w t(u \mid u+v) \geq$ $d\left(C_{2}\right)$.

For instance, by combining the pure codes $[[14,8,3]]$ and $[[14,0,6]]$, we obtain an $[[28,8,6]]$ quantum stabilizer code.

\subsection{Quantum Stabilizer Codes from Nearly Self-orthogonal Quaternary Linear Codes}

In this Section, we introduce another method of constructing new quantum codes. The construction is based on construction $\mathrm{X}$ and its variants [13], and we use linear codes as our source code. So, we will apply the Hermitian inner product which is equivalent to the symplectic inner product in the case code is linear 2.1.2. We will denote the Hermitian inner product of $u, v \in \mathbb{F}_{4}^{n}$ by $\langle u, v\rangle$.

For $u \in \mathbb{F}_{4}^{n}$, note that the norm of $u$ is $\|u\|=<u, u>=\sum_{i=0}^{n} u_{i}{ }^{3}$. If $u_{i} \neq 0$ then $u_{i}{ }^{3}=1$, so $w t(u)=\|u\|$ and by the proof of Theorem 2.1.3 we see that

$$
\|u+v\|=\|u\|+\|v\|+\operatorname{Tr}(<u, v>) .
$$

A set $S \subseteq \mathbb{F}_{4}^{n}$ is called an orthonormal set if $\langle u, v\rangle=0$ for $u \neq v \in S$ and $\|u\|=1$ for all $u \in S$.

Proposition 2.3.10 Let $D$ be a linear subspace of $\mathbb{F}_{4}^{n}$ and the set $M$ be a basis for $D \cap D^{\perp}$. Then there exists a set $B$ which is orthonormal and $M \cup B$ is a basis for $D$.

Proof: First, suppose that $R$ is a subspace of $\mathbb{F}_{4}^{n}$ and there exist $u, v \in R$ such that $<u, v>\neq 0$. Then, for $\gamma \in \mathbb{F}_{4}^{*}$,

$$
\|\gamma u+v\|=\|\gamma u\|+\|v\|+\operatorname{Tr}(\gamma<u, v>) .
$$

So we can find $\gamma$ such that $\|\gamma u+v\|=1$. Now, let $W$ be a subspace of $\mathbb{F}_{4}^{n}$ such that

$$
D=\left(D \cap D^{\perp}\right) \oplus W .
$$

We will assume that $W$ is not self-orthogonal, since otherwise $D=\left(D \cap D^{\perp}\right)$ and in this case we can turn $M$ into an orthonormal basis as described above. Let $r:=\operatorname{dim}(W)$. For each $0 \leq i \leq r$ we will construct a set $S_{i}$ which is an orthonormal basis of $T_{i}$, where $T_{i}$ is a subspace of $W$ with

$$
W=T_{i} \oplus\left(T_{i}^{\perp} \cap W\right) .
$$

The steps are iterative. Take $S_{0}=\emptyset$ and suppose that for some $0 \leq i<r$ there is an orthonormal basis $S_{i}$ of $T_{i}$, where $\operatorname{dim}\left(T_{i}\right)=i$ and it satisfies (2.2). Let $u$ be a nonzero vector in $T_{i}{ }^{\perp} \cap W$. Then there exists a vector $v \in T_{i}{ }^{\perp} \cap W$ such that $\langle u, v\rangle \neq 0$. 
Note that there exists such $v$, because $W$ is not self-orthogonal. According to the first paragraph of the proof, there exists $\gamma \in \mathbb{F}_{4}^{*}$ such that $\|\gamma u+v\|=1$. Let $z=\gamma u+v$. Then the set $S_{i+1}=S_{i} \cup\{z\}$ is an orthonormal set and $z \notin T_{i}$, hence $\operatorname{dim}\left(T_{i+1}\right)=i+1$. Now, we show that

$$
W=T_{i+1} \oplus\left(T_{i+1}^{\perp} \cap W\right) .
$$

First we prove that $T_{i+1} \cap\left(T_{i+1}^{\perp} \cap W\right)=\{0\}$. Suppose $x \in T_{i+1} \cap\left(T_{i+1} \perp \cap W\right)$. Since $x \in T_{i+1}$, we have $x=y+\alpha z$ where $y \in T_{i}$ and $\alpha \in \mathbb{F}_{4}$. Moreover, since $x=y+\alpha z \in T_{i+1}^{\perp}$ for all $w \in T_{i}$ and $\beta \in \mathbb{F}_{4}$, we have

$0=<y+\alpha z, w+\beta z>=<y, w>+\bar{\beta}<y, z>+\alpha<z, w>+\alpha \bar{\beta}\|z\|=<y, w>+\alpha \bar{\beta}$.

Therefore, $\alpha=0$, because otherwise we can find a $\beta \in \mathbb{F}_{4}$ such that $<y, w>+\alpha \bar{\beta} \neq 0$, which is a contradiction. So, $\langle y, w\rangle=0$ and since $w \in T_{i}$, we conclude that $y \in T_{i}{ }^{\perp}$. Moreover, $x=y$ also belongs to $T_{i}$. Hence, $x \in T_{i} \cap T_{i}{ }^{\perp}=\{0\}$ and it shows $T_{i+1} \cap\left(T_{i+1}{ }^{\perp} \cap W\right)=\{0\}$. Next we prove that $W=T_{i+1}+\left(T_{i+1}{ }^{\perp} \cap W\right)$. Let $w \in W$. Since $W=T_{i} \oplus\left(T_{i}{ }^{\perp} \cap W\right)$ there are $x \in T_{i}$ and $y \in T_{i}^{\perp} \cap W$ such that $w=x+y$. Now, let $x^{\prime}=x+<y, z>z$ and $y^{\prime}=y-<y, z>z$. Obviously $x^{\prime} \in T_{i+1}$ and for any $s=u+\alpha z \in T_{i+1}$ with $u \in T_{i}, \alpha \in \mathbb{F}_{4}$. We have

$$
\begin{aligned}
<y^{\prime}, s> & =<y-<y, z>z, u+\alpha z> \\
= & <y, u>+\bar{\alpha}<y, z>-<y, z><z, u>-\bar{\alpha}<y, z>\|z\| \\
= & \bar{\alpha}(<y, z>-<y, z>)=0 .
\end{aligned}
$$

Therefore, $y^{\prime} \in T_{i}{ }^{\perp} \cap W$ and $x^{\prime}+y^{\prime}=x+y=w$. So (2.2) implies (2.3) and by repeating this approach we can achieve the desired goal.

Now we state the main theorem of this Section.

Theorem 2.3.11 Suppose that $C$ is an $[n, k]_{4}$ linear code and $e:=n-k-\operatorname{dim}(C \cap$ $\left.C^{\perp_{s}}\right)$. Then there exist a quantum code with parameters $[[n+e, 2 k-n+e, d]]$ with $d \geq \min \left\{d(C), d\left(C+C^{\perp_{s}}\right)+1\right\}$.

Proof: First, note that $\operatorname{dim}\left(C^{\perp_{s}}\right)=n-k$ and we have $\operatorname{dim}\left(C+C^{\perp_{s}}\right)=\operatorname{dim}(C)+$ $\operatorname{dim}\left(C^{\perp_{s}}\right)-\operatorname{dim}\left(C \cap C^{\perp_{s}}\right)$. So $e=\operatorname{dim}\left(C+C^{\perp_{s}}\right)-\operatorname{dim}(C)$. Let $s:=\operatorname{dim}\left(C \cap C^{\perp_{s}}\right)$. Consider the block matrix $G$

$$
G=\left[\begin{array}{cc}
M_{s \times n} & 0_{s \times e} \\
A_{(n-e-2 s) \times n} & 0_{(n-e-2 s) \times e} \\
B_{e \times n} & I_{e \times e}
\end{array}\right],
$$

where the indices show the size of blocks, and $0, I$ are the zero and identity matrices, respectively. We denote the rows of a matrix $P$ by $r(P)$. The matrix $G$ is constructed such that $r(M)$ is a basis for $C \cap C^{\perp_{s}}, r(M) \cup r(A)$ is a basis for $C$ and $r(M) \cup r(B)$ is a basis for $C^{\perp_{s}}$. Note that according to Proposition 2.3.10 we can choose $B$ as an orthonormal set and $r(M) \cup r(A) \cup r(B)$ is a basis for $\left(C+C^{\perp_{s}}\right)$.

Let $E$ be the linear code of length $n+e$ generated by the matrix $G$. Now consider the matrix $T$

$$
T=\left[\begin{array}{ll}
M_{s \times n} & 0_{s \times e} \\
B_{e \times n} & I_{e \times e}
\end{array}\right] .
$$


By construction, each row of $T$ is orthogonal to every row of $G$. So $r(T) \subseteq E^{\perp_{s}}$. Moreover, since the vectors in $r(T)$ are self-orthogonal and $\operatorname{dim}\left(E^{\perp_{s}}\right)=n+e-(n-s)=$ $e+s$, we conclude that $r(T)$ is a basis for $E^{\perp_{s}}$ and consequently $E^{\perp_{s}} \subseteq E$. So $E^{\perp_{s}}$ generates a self-orthogonal code. Since $E^{\perp_{s}}$ has $2^{2(s+e)}$ elements, by Definition 2.1.1 it yields a quantum stabilizer code of dimension $n+e-2(s+e)=2 k-n+e$.

Now let $x$ be a nonzero vector in $E$. So $x$ is linear combination of rows of $G$ and we can write $x=\left(x_{1} \mid x_{2}\right)$ where $x_{1} \in \mathbb{F}_{4}^{n}$ and $x_{2} \in \mathbb{F}_{4}^{e}$. If there is no vectors from the last $e$ rows of $G$, then $x_{1} \in C$ and $x_{2}=0$ and therefore $w t(x) \geq d(C)$. Otherwise, some of the vectors in the last $e$ rows of $G$ are included in the linear combination and $w t\left(x_{1}\right) \geq d\left(C+C^{\perp_{s}}\right)$ and $w t\left(x_{2}\right) \geq 1$. Hence, $w t(x) \geq d\left(C+C^{\perp_{s}}\right)+1$. This completes the proof.

In Theorem 2.3.11, the construction is based on a linear source code. So it is possible to choose our source code from any class linear codes. For example, we can replace linear codes with cyclic codes or $2-\mathrm{D}$ cyclic codes. We will explain the cyclic construction in this Section and 2-D cyclic code construction in the next Chapter. First, we need the following proposition.

Proposition 2.3.12 Suppose $C$ is a quaternary cyclic code with length $n$, where $n$ is an odd number. Let $Z$ be the defining set of the code $C$. Then $\operatorname{dim}\left(C^{\perp_{s}}\right)-\operatorname{dim}(C \cap$ $\left.C^{\perp_{s}}\right)=|Z \cap-2 Z|$, where $-2 Z:=\{-2 z(\bmod n) \mid z \in Z\}$.

Proof: Let $\beta$ be a primitive $n$th root of unity, $\bar{Z}:=Z_{n}-Z$ and

$$
g(x)=\prod_{k \in Z}\left(x-\beta^{k}\right)
$$

be the generator polynomial of code $C$. Then its Hermitian (symplectic) dual generator will be

$$
h(x)=\prod_{k \in-2 \bar{Z}}\left(x-\beta^{k}\right),
$$

and the generator polynomial of $C \cap C^{\perp_{s}}$ is

$$
k(x)=\prod_{k \in Z \cup-2 \bar{Z}}\left(x-\beta^{k}\right) .
$$

Then,

$$
\begin{aligned}
\operatorname{dim}\left(C^{\perp_{s}}\right)-\operatorname{dim}\left(C \cap C^{\perp_{s}}\right) & =(n-|-2 \bar{Z}|)-(n-|Z \cup-2 \bar{Z}|) \\
& =|Z \cup-2 \bar{Z}|-|-2 \bar{Z}| \\
& =|Z-2 \bar{Z}|=|Z \cap-2 Z| .
\end{aligned}
$$

Note that if $n$ is an odd number which is divisible by 3 , then $\{0\},\left\{\frac{n}{3}\right\}$, and $\left\{\frac{2 n}{3}\right\}$ have singleton cyclotomic cosets modulo $n$ and are closed under multiplication by -2 modulo $n$. So if $C$ is a cyclic code with defining set $Z \subseteq\left\{0, \frac{n}{3}, \frac{2 n}{3}\right\}$, then by Proposition 2.3.12, $\operatorname{dim}\left(C^{\perp_{s}}\right)-\operatorname{dim}\left(C \cap C^{\perp_{s}}\right)=|Z|$.

Theorem 2.3.13 Suppose that $n$ is an odd integer which is divisible by 3 and let $C$ be an $[n, k]_{4}$ cyclic code with the defining set $Z$ where $Z \cap-2 Z \subseteq\left\{0, \frac{n}{3}, \frac{2 n}{3}\right\}$. Then there exists a quantum code with parameters $[[n+e, 2 k-n+e, d]]$ with 


$$
d \geq \min \left\{d\left(C_{U}\right)+|U|\right\}
$$

where $U \subseteq Z \cap-2 Z, C_{U}$ is a cyclic code with defining set $Z \backslash U$, and $e=|Z \cap-2 Z|$.

Proof: Let $r:=\frac{n}{3}$ and $\beta$ be a primitive $n$th root of unity in $\mathbb{F}_{4^{m}}$, where $m$ is order of 4 modulo $n$, then $\omega=\beta^{r}$ is a primitive cube root of unity in $\mathbb{F}_{4}$. We define

$$
b_{t}(x):=\frac{x^{n}-1}{x-\beta^{t r}}=\frac{x^{n}-1}{x-\omega^{t}}=\sum_{i=0}^{r-1} x^{3 i+2}+\omega^{t} x^{3 i+1}+\omega^{2 t} x^{3 i}
$$

where $t \in\{0,1,2\}$. So, we can consider each $b_{t}$ as a codeword in the form $\left(a_{0}, a_{1}, \cdots, a_{n-1}\right)$ in $\mathbb{F}_{4}^{n}$. Since $n$ is odd, $\left\langle b_{t}, b_{t}\right\rangle=n \equiv 1 \bmod 2$. By construction, if $t \neq t^{\prime}$ then $<b_{t}, b_{t^{\prime}}>=0$. So the set $\left\{b_{1}, b_{2}, b_{3}\right\}$ is an orthonormal set. Now, we can use the construction in Theorem 2.3.11. The difference here is that in the last $e$ rows we use $b_{i}$ 's. Therefore, using Proposition 2.3.12 and Theorem 2.3.11, we have the matrix $G$

$$
G=\left[\begin{array}{cc}
M_{s \times n} & 0_{s \times e} \\
A_{(n-e-2 s) \times n} & 0_{(n-e-2 s) \times e} \\
B_{e \times n} & I_{e \times e}
\end{array}\right],
$$

where $r(M)$ is a basis for $C \cap C^{\perp_{s}}, r(M) \cup r(A)$ is a basis for $C$, and $r(M) \cup r(B)$ is a basis for $C^{\perp_{s}}$. Let $E$ be the code generated by the matrix $G$. Clearly, the code generated by the matrix

$$
T=\left[\begin{array}{cc}
M_{s \times n} & 0_{s \times e} \\
B_{e \times n} & I_{e \times e}
\end{array}\right]
$$

is $E^{\perp_{s}}$ and therefore $E^{\perp_{s}} \subseteq E$. Let $x$ be a nonzero codeword in $E$. We can write $x=\left(x_{1} \mid x_{2}\right)$ where $x_{1} \in \mathbb{F}_{4}^{n}$ and $x_{2} \in \mathbb{F}_{4}^{e}$. If no row from $r(B)$ occurs in the linear combination of rows defining $x$, then $w t(x) \geq d(C)$. If there are some rows of $r(B)$ in the linear combination, then $w t(x) \geq d(\operatorname{Span}(C, U)+|U|)$, where $U=\left\{b_{i}\right\}$ for all $b_{i}$ occurring in the linear combination, and $\operatorname{Span}\left(C,\left\{b_{i}\right\}\right)$ is exactly the cyclic code $C_{U}$. Therefore, $w t(x) \geq \min \left\{d\left(C_{U}\right)+|U|\right\}$, where $U \subseteq Z \cap-2 Z$.

\subsection{Non-binary Quantum Stabilizer Codes}

Denote by $\mathbb{F}_{p^{m}}$ the finite field of $p^{m}$ elements, where $p$ is a prime number and $m$ is a positive integer. Let $\operatorname{Tr}: \mathbb{F}_{p^{m}} \rightarrow \mathbb{F}_{p}$ be the standard trace function which is an $\mathbb{F}_{p}$ linear map and defined by $\operatorname{Tr}(a)=\sum_{i=0}^{m-1} a^{p^{i}}$. Let $a, b \in \mathbb{F}_{p^{m}}^{n}$, and consider the Euclidean inner product of $a$ and $b$

$$
<a, b>=\sum_{i=1}^{n} a_{i} b_{i}
$$

Definition 2.4.2 Let $(a, b),\left(a^{\prime}, b^{\prime}\right) \in \mathbb{F}_{p^{m}}^{2 n}$. We define the symplectic inner product of $(a, b)$ and $\left(a^{\prime}, b^{\prime}\right)$ as 


$$
(a, b) *\left(a^{\prime}, b^{\prime}\right)=\operatorname{Tr}\left(<a, b^{\prime}>-<a^{\prime}, b>\right) .
$$

Also, the symplectic weight of $(a, b)$ will be denoted by

$$
\operatorname{swt}((a, b))=\left|\left\{1 \leq k \leq n \mid\left(a_{k}, b_{k}\right) \neq(0,0)\right\}\right| .
$$

For an $\mathbb{F}_{p}$ additive code $C \subseteq \mathbb{F}_{p^{m}}^{2 n}$ we denote the symplectic dual of $C$ with

$$
C^{\perp_{s}}=\left\{(c, d) \in \mathbb{F}_{p^{m}}^{2 n} \mid(a, b) *(c, d)=0 \text { for all }(a, b) \in C\right\} .
$$

Remark 2.4.1 If there exists an additive code $C \subseteq \mathbb{F}_{q}^{2 n}$ such that $|C|=q^{n-k}, C \subseteq$ $C^{\perp_{s}}$, and $d=\operatorname{swt}\left(C^{\perp_{s}} \backslash C\right.$, then an $[[n, k, d]]_{q}$ quantum stabilizer code exists.

Similar to the binary case if $k=0$, which implies $C=C^{\perp_{s}}$, then we define $d=$ $\min \{\operatorname{swt}(u) \mid 0 \neq u \in C\}$. Let $u, v$ be elements of $\mathbb{F}_{p^{2}}^{n}$, then the Hermitian inner product of $u$ and $v$ is defined as follow

$$
<u, v>=\sum_{i=1}^{n} a_{i} \overline{b_{i}}
$$

where $\bar{x}=x^{p}$. The following analogue of Theorem 2.1.2 hold for characteristic $p$ too.

Theorem 2.4.14 An $\mathbb{F}_{p^{2}}$ linear code $C$ is self-orthogonal with respect to the symplectic inner product if and only if it is self-orthogonal with respect to the Hermitian inner product.

Theorem 2.4.15 If there exists an $\mathbb{F}_{p^{2}}$-linear $[n, k, d]_{p^{2}}$ code $C$ such that $C^{\perp_{h}} \subseteq C$, then there exists an $[[n, 2 k-n, d]]$ quantum stabilizer code where $d=\operatorname{swt}\left(C \backslash C^{\perp_{h}}\right)$

For proofs of Theorem 2.4.14 and 2.4.15, we refer to [1].

Now, we extend the construction $X$ to obtain quantum stabilizer codes over finite fields of order $p^{2}$. Almost all of the results will be generalizations of the results in Section 2.3, so we skip some of the proofs. For more information we refer to [5]

Lemma 2.4.16 Let $D$ be a linear subspace of $\mathbb{F}_{p^{2}}^{n}$ and $M$ be a basis for $D \cap D^{\perp_{h}}$. Then there exists an orthonormal set $B$ such that $M \cup B$ is a basis for $D$.

The proof is a generalization of the proof of Theorem2.3.10 and therefore we omit it. In the rest of this chapter we assume that $p$ is a prime number such that $p-1=4 k$ for some integer $k$.

Theorem 2.4.17 Suppose that $C$ is an $\mathbb{F}_{p^{2}}$ linear code with parameters $[n, k]_{p^{2}}$. Let $e=n-k-\operatorname{dim}\left(C \cap C^{\perp_{h}}\right)$. Then there exists an $[[n+e, 2 k-n+e, d]]_{p^{2}}$ quantum code where $d \geq \min \left\{d(C), d\left(C+C^{\perp_{h}}\right)+1\right\}$.

Proof: First, we prove that there exist a nonzero element $\gamma$ in $\mathbb{F}_{p^{2}}$ such that $\gamma^{2}+1=0$. We know that $\mathbb{F}_{p^{2}}^{*}$ is a cyclic group. Let $\beta$ be a generator for this group. Then, $\beta^{p^{2}-1}=1$. Moreover, $p^{2}-1=4 k$, where $k$ is an integer. Therefore, $\beta^{\frac{p^{2}-1}{2}}=-1$. Now, consider the matrix

$$
G=\left[\begin{array}{cc}
M_{s \times n} & 0_{s \times e} \\
A_{(n-e-2 s) \times n} & 0_{(n-e-2 s) \times e} \\
B_{e \times n} & \beta^{\frac{p-1}{4}} I_{e \times e}
\end{array}\right],
$$


where $s=\operatorname{dim}\left(C \cap C^{\perp_{h}}\right)$. The size of each block is determined by the index, and 0 and $I$ denote the zero matrix and identity matrix, respectively. Here, for matrix $P$, $r(P)$ denores the rows of matrix $P$. In $G, r(M)$ is a basis for $C \cap C^{\perp_{h}}, r(M) \cup r(A)$ is a basis for $C, r(M) \cup r(B)$ is a basis for $C^{\perp_{h}}$, and finally $r(B)$ is the orthonormal set which is obtained by Lemma 2.4.16.

Let $E$ be the linear code with the generator matrix $G$. Now, consider the matrix $S$ which is defined as follows:

$$
S=\left[\begin{array}{cc}
M_{s \times n} & 0_{s \times e} \\
B_{e \times n} & \beta^{\frac{p-1}{4}} I_{e \times e}
\end{array}\right]
$$

By construction, each of the first $s$ vectors in $r(S)$ is orthogonal to each row of $G$. Also, if $v=\left(v_{1}, v_{2}\right)$ is one of the vectors from the last $e$ rows of $S$, then $v$ is orthogonal to first $n-e-s$ rows of $G$. Now we consider the inner product of $v=\left(v_{1}, v_{2}\right)$ and $u=\left(u_{1}, u_{2}\right)$, where $u, v$ are in the last $e$ rows of $S$. If $u \neq v$, then by construction $<u, v>=0$. Otherwise, if $u=v$,

$$
\begin{aligned}
<u, v> & =\left\langle v, v>=\|v\|=\sum_{i=1}^{n+e} v_{i}^{p+1}=\sum_{i=1}^{n} v_{i}^{p+1}+\sum_{i=n+1}^{n+e} v_{i}^{p+1}\right. \\
& =1+\beta^{\frac{p-1}{4}}=1+\beta^{\frac{(p-1)(p+1)}{4}}=1+\beta^{\frac{p^{2}-1}{4}}=1-1=0 .
\end{aligned}
$$

So, each vector from $S$ belongs to $E^{\perp_{h}}$. Rest of the proof is similar to the proof of Theorem 2.3.11

Next, we will explain another method of constructing quantum codes using cyclic codes. First, we need the following Lemma.

Lemma 2.4.18 Let $C$ be a cyclic code over $\mathbb{F}_{p^{2}}$ with the defining set $Z \subseteq \mathbb{Z}_{n}$. Then, $\operatorname{dim}\left(C^{\perp_{h}}\right)-\operatorname{dim}\left(C \cap C^{\perp_{h}}\right)=|Z \cap-p Z|$.

Proof: Similar to Proposition 2.3.12.

Theorem 2.4.19 Assume that $n$ is divisible by $p^{2}-1$ and $C$ be an $[n, k]_{p^{2}}$ cyclic code with the defining set $Z$ where $Z \cap-p Z \subseteq T=\left\{\frac{n k}{p^{2}-1} \mid 1 \leq k \leq p^{2}-1\right\}$. If $e=|Z \cap-p Z|$, then there exists an $[[n+e, 2 k-n+e, d]]_{p^{2}}$ quantum code with $d \geq$ $\min \left\{d(C), d\left(C_{u}\right)+1, d\left(C+C^{\perp_{h}}\right)+2\right\}$, where $u \in Z \cap-p Z$ and $C_{u}$ is a cyclic code with defining set $Z \backslash\{u\}$.

Proof: First we show that each element in $T$ has a singleton cyclotomic coset. Assume that $1 \leq k \leq p^{2}-1$. Then

$$
\frac{n k}{p^{2}-1} p^{2}=\frac{p^{2} n k}{p^{2}-1}=\frac{p^{2} n k-n k+n k}{p^{2}-1}=n k+\frac{n k}{p^{2}-1} \equiv \frac{n k}{p^{2}-1} \bmod n .
$$

Next, let $q=p^{2}-1, n=\left(p^{2}-1\right) l=q l$ and $\omega$ be a $\left(p^{2}-1\right)$ th root of unity. Consider the polynomials

$$
b_{t}(x)=\frac{x^{n}-1}{x-\omega^{t}}=\sum_{i=1}^{l-1}\left(x^{q i+q-1}+\omega^{t} x^{q i+q-2}+\cdots+\omega^{(q-1) t} x^{q i}\right),
$$

for $0 \leq t \leq l$. We show the corresponding codewords with $b_{t}$. Now, we show that the set $\left\{b_{j} \mid 0 \leq j \leq l\right\}$ is an orthonormal set. This is because 


$$
<b_{u}, b_{v}>=q \sum_{i=0}^{l-1} \omega^{i(u+v p)}=q \sum_{i=0}^{l-1} \omega^{i(u-v p)}=\left\{\begin{array}{ll}
q l & u=v \\
0 & u \neq v
\end{array} .\right.
$$

Rest of the proof is similar to the proof of Theorem 2.3.13. 


\section{CHAPTER 3}

\section{New Constructions of Quantum Stabilizer Codes}

In Chapter 2, we presented some methods of constructing new quantum codes using classical codes or extending primary quantum codes. In this Chapter, first we will introduce 2-D cyclic quantum stabilizer codes and construct new quantum codes based on classical 2-D cyclic codes. Next, we introduce a method of constructing quantum codes over $\mathbb{F}_{p^{2}}$ by using arbitrary classical additive codes over $\mathbb{F}_{p^{2}}$, where $p$ is an odd prime number.

\subsection{2-D Cyclic Quantum Stabilizer Codes}

By Theorem 2.4.15, for an $[n, k]_{p^{2}}$ linear code $C$ such that $C^{\perp_{h}} \subseteq C$ we can find a quantum code with parameters $[[n, 2 k-n, d]]$, where $d=\min \left\{w t(u) \mid u \in C \backslash C^{\perp_{h}}\right\}$.

Definition 3.1.1 Suppose there exists a 2-D cyclic code $C$ with the parameters $\left[n_{1} \times n_{2}, k, d\right]_{p^{2}}$ such that $C^{\perp_{h}} \subseteq C$. Then we call $C$ a $2-D$ cyclic quantum code.

Now, let $C=I(U) /<x^{n_{1}}-1, y^{n_{2}}-1>$ be a $2-\mathrm{D}$ cyclic code with the zero set $U$. Then by Theorem 1.5.15, we can find $G=\left\{p_{0}(x, y), p_{1}(x, y), \cdots, p_{n_{2}-1}(x, y)\right\}$ which is the set of the generator polynomials for $C$. Then $C$ is a 2-D cyclic quantum code if and only if

$$
p_{i}(x, y) \cdot \overline{p_{j}(x, y)} \equiv 0 \bmod <x^{n_{1}}-1, y^{n_{2}}-1>\text { for all } p_{i}(x, y), p_{j}(x, y) \in G .
$$

So we have the following Proposition.

Proposition 3.1.1 Suppose that $C=I(U) /<x^{n_{1}}-1, y^{n_{2}}-1>$ is a $2-D$ cyclic code which is generated by the polynomials $p_{0}(x, y), p_{1}(x, y), \cdots, p_{n_{2}-1}(x, y)$, and $p_{0}^{0}(x) \cdot \overline{p_{0}^{0}(x)} \equiv$ $0 \bmod <x^{n_{1}}-1>$. Then $C$ is a 2-D cyclic quantum code.

Proof: By Proposition 1.5.14 $p_{0}^{0}(x) \mid p_{i}^{j}(x)$ for all $0 \leq i, j \leq n_{2}-1$. Then $p_{i}^{j}(x) \cdot \overline{p_{i}^{j}(x)} \equiv$ $0 \bmod <x^{n_{1}}-1>$ for all $0 \leq i, j \leq n_{2}-1$. It means that for all $0 \leq i \leq n_{2}-1$ we have

$$
p_{i}(x, y) \cdot \overline{p_{i}(x, y)} \equiv 0 \bmod <x^{n_{1}}-1>\text {. }
$$

Therefore, $C \subseteq C^{\perp_{h}}$, which means that $C$ is a 2-D cyclic quantum code.

Example 3.1.2 Consider the quaternary 2-D cyclic code $C$ with the parameters $[15,8]_{2}$ and the generator matrix 


$$
G=\left[\begin{array}{ccccccccccccccc}
1 & 0 & 1 & w^{2} & w^{2} & 0 & 0 & 0 & 0 & 0 & w & 0 & w & 1 & 1 \\
w & 0 & w & 1 & 1 & 0 & 0 & 0 & 0 & 0 & w^{2} & 0 & w^{2} & w & w \\
0 & 1 & w^{2} & w^{2} & 1 & 0 & 0 & 0 & 0 & 0 & 0 & w & 1 & 1 & w \\
0 & w & 1 & 1 & w & 0 & 0 & 0 & 0 & 0 & 0 & w^{2} & w & w & w^{2} \\
0 & 0 & 0 & 0 & 0 & 1 & 0 & 1 & w^{2} & w^{2} & w^{2} & 0 & w^{2} & w & w \\
0 & 0 & 0 & 0 & 0 & w & 0 & w & 1 & 1 & 1 & 0 & 1 & w^{2} & w^{2} \\
0 & 0 & 0 & 0 & 0 & 0 & 1 & w^{2} & w^{2} & 1 & 0 & w^{2} & w & w & w^{2} \\
0 & 0 & 0 & 0 & 0 & 0 & w & 1 & 1 & w & 0 & 1 & w^{2} & w^{2} & 1
\end{array}\right],
$$

where $w \neq 0,1$ is an element of $\mathbb{F}_{4}$. The code is self-orthogonal with respect to the Hermitian inner product and consequently we can find a [[15,7]] quantum code. Using Magma, we can see that $\min \left\{w t(u) \mid u \in C^{\perp_{h}} \backslash C\right\}=3$. Therefore, $C$ is an $[[15,7,3]]$ quantum code. According to the best known quantum code's table [8], this code reaches the optimum minimum distance.

\subsection{New Quantum Stabilizer Codes Construction Using 2-D Cyclic Codes}

Now, we introduce a new method of constructing quantum stabilizer codes which is based on construction X. Our main aim is to replace the code $C$ in Theorem 2.4.17 with a 2-D cyclic code. In this Section, we assume that $p$ is a prime number such that $p=4 k+1$ for some integer $k$.

Lemma 3.2.3 Let $n_{1}, n_{2}$ be two positive integers such that $p^{2}-1$ divides both $n_{1}$ and $n_{2}$. Then the $\mathbb{F}_{p^{2}}$ conjugacy class of $\left(\alpha_{1}{ }^{i}, \alpha_{2}{ }^{j}\right)$ is a singleton, if $i \in T_{1}=\left\{\frac{n_{1} k}{p^{2}-1} \mid k \in\right.$ $\left.\left\{1,2, \cdots, p^{2}-1\right\}\right\}$ and $j \in T_{2}=\left\{\frac{n_{2} k}{p^{2}-1} \mid k \in\left\{1,2, \cdots, p^{2}-1\right\}\right\}$.

Proof: It is enough to show that $\left(\alpha_{1}{ }^{i p^{2}}, \alpha_{2}{ }^{j p^{2}}\right)=\left(\alpha_{1}{ }^{i}, \alpha_{2}{ }^{j}\right)$ for all $i \in T_{1}$ and $j \in T_{2}$. Suppose $i=\frac{n_{1} k}{p^{2}-1}, j=\frac{n_{2} k^{\prime}}{p^{2}-1}$ we have:

$$
\begin{aligned}
& \left(\alpha_{1}^{i p^{2}}, \alpha_{2}^{j p^{2}}\right)=\left(\alpha_{1}^{\frac{n_{1} k}{p^{2}-1} p^{2}}, \alpha_{2}^{\frac{n_{2} k^{\prime}}{p^{2}-1} p^{2}}\right)=\left(\alpha_{1}^{\frac{p^{2} \cdot n_{1} \cdot k}{p^{2}-1}}, \alpha_{2}^{\frac{p^{2} n_{2} k^{\prime}}{p^{2}-1}}\right) \\
& =\left(\alpha_{1}^{\frac{p^{2} \cdot n_{1} \cdot k+n_{1} \cdot k-n_{1} \cdot k}{p^{2}-1}}, \alpha_{2} \frac{p^{2} n_{2} k^{\prime}+n_{2} \cdot k^{\prime}-n_{2} \cdot k^{\prime}}{p^{2}-1}\right)=\left(\alpha_{1}{ }^{n_{1} k} \alpha_{1}{ }^{\frac{n_{1} k}{p^{2}-1} p^{2}}, \alpha_{2}{ }^{n_{2} k^{\prime}} \alpha_{2} \frac{n_{2} k^{\prime}}{p^{2}-1} p^{2}\right) \\
& =\left(\alpha_{1}{ }^{i}, \alpha_{2}{ }^{j}\right) \text {. }
\end{aligned}
$$

Theorem 3.2.4 Suppose that $n_{1}$ and $n_{2}$ are divisible by $p^{2}-1$ and let $C$ be an $\left[n_{1} \times n_{2}, k\right]_{p^{2}} 2-D$ cyclic code with the zero set $U$ such that $U \cap U^{-p} \subseteq\left\{\left(\alpha_{1}{ }^{i}, \alpha_{2}{ }^{j}\right) \mid(i, j) \in\right.$ $\left.T_{1} \times T_{2}\right\}$, where $T_{1}, T_{2}$ are defined as in Lemma 3.2.3. If $e=\left|U \cap U^{-p}\right|$, then there exists an $\left[\left[n_{1} \times n_{2}+e, 2 k-n_{1} \times n_{2}+e, d\right]\right]_{p}$ quantum code with $d \geq \min \left\{d(C), d\left(C_{V}\right)+r\right\}$, where the minimum is taken over all the 2 - $D$ cyclic codes $C_{V}$ with the zero set $V \subseteq$ $U \cap U^{-p}$ and $r=|V|$. 
Proof: We prove the theorem in two steps. First step: Let $q=p^{2}-1, n_{1}=q h, n_{2}=$ $q h^{\prime}$, and $\omega$ be a $\left(p^{2}-1\right)$ th root of unity. Then, we claim that the set of codewords corresponding to the elements of the set $T=\left\{b_{i, j}=\frac{x^{n_{1}}-1}{x-\omega^{i}} \times \frac{y^{n_{2}-1}}{y-\omega^{j}} \mid 0 \leq i \leq h, 0 \leq j \leq\right.$ $\left.h^{\prime}\right\}$ is an orthonormal set.

Let $b_{i, j} \in T$ be of the form

$$
\begin{aligned}
b_{i, j}= & \left(\sum_{s=0}^{h-1}\left(x^{q s+q-1}+\omega^{i} x^{q s+q-2}+\cdots+\omega^{(q-1) i} x^{q s}\right)\right) \times \\
& \left(\sum_{k=0}^{h^{\prime}-1}\left(y^{q k+q-1}+\omega^{j} y^{q k+q-2}+\cdots+\omega^{(q-1) j} x^{q k}\right)\right) .
\end{aligned}
$$

We can express all the coefficients of the product

$$
\left(x^{q s+q-1}+\omega^{i} x^{q s+q-2}+\cdots+\omega^{(q-1) i} x^{q s}\right)\left(y^{q k+q-1}+\omega^{j} y^{q k+q-2}+\cdots+\omega^{(q-1) j} x^{q k}\right)
$$

as a $q \times q$ matrix

$$
W_{i, j}=\left[\begin{array}{ccccc}
\omega^{(q-1) j} \omega^{(q-1) i} & \omega^{(q-1) j} \omega^{(q-2) i} & \omega^{(q-1) j} \omega^{(q-3) i} & \cdots & \omega^{(q-1) j} \\
\omega^{(q-2) j} \omega^{(q-1) i} & \omega^{(q-2) j} \omega^{(q-2) i} & \omega^{(q-2) j} \omega^{(q-3) i} & \cdots & \omega^{(q-2) j} \\
\vdots & \vdots & \vdots & \cdots & \vdots \\
\omega^{(q-1) i} & \omega^{(q-2) i} & \omega^{(q-3) i} & \cdots & 1
\end{array}\right] .
$$

So we can represent each $b_{i, j}$ as a matrix with $h h^{\prime}$ blocks, where all of the blocks are $W_{i, j}$. We will show that

$$
<W_{i, j}, W_{i^{\prime}, j^{\prime}}>\left\{\begin{array}{ll}
=0, & \text { if } W_{i, j} \neq W_{i^{\prime}, j^{\prime}} \\
\neq 0, & \text { if } W_{i, j}=W_{i^{\prime}, j^{\prime}}
\end{array},\right.
$$

and consequently

$$
<b_{i, j}, b_{i^{\prime}, j^{\prime}}>=h . h^{\prime}<W_{i, j}, W_{i^{\prime}, j^{\prime}}>\left\{\begin{array}{ll}
=0, & \text { if } b_{i, j} \neq b_{i^{\prime}, j^{\prime}} \\
\neq 0, & \text { if } b_{i, j}=b_{i^{\prime}, j^{\prime}}
\end{array},\right.
$$

which completes the proof.

Let $\omega^{(q-t) j}\left(\omega^{(q-1) i}, \omega^{(q-2) i}, \cdots, \omega^{i}\right)$ and $\omega^{(q-t) j^{\prime}}\left(\omega^{(q-1) i^{\prime}}, \omega^{(q-2) i^{\prime}}, \cdots, \omega^{i^{\prime}}\right)$ be the $t$-th rows of $W_{i, j}$ and $W_{i^{\prime}, j^{\prime}}$. Then

$$
\begin{aligned}
& \omega^{(q-t) j}\left(\omega^{(q-1) i}, \omega^{(q-2) i}, \cdots, \omega^{i}\right) \cdot \overline{\omega^{(q-t) j^{\prime}}\left(\omega^{(q-1) i^{\prime}}, \omega^{(q-2) i^{\prime}}, \cdots, \omega^{i^{\prime}}\right)} \\
= & \left(\omega^{(q-t) j}\right)\left(\omega^{(q-1) i}, \omega^{(q-2) i}, \cdots, \omega^{i}\right) \cdot\left(\omega^{(q-t) j^{\prime} p}\right)\left(\omega^{(q-1) i^{\prime} p}, \omega^{(q-2) i^{\prime} p}, \cdots, \omega^{i^{\prime} p}\right) .
\end{aligned}
$$

Now since the conjugacy class of $b_{i, j}=\left(\omega^{i^{\prime}}, \omega^{j^{\prime}}\right)$ is a singleton class and $U \cap U^{-p} \subseteq$ $\left\{\left(\alpha_{1}{ }^{i}, \alpha_{2}{ }^{j}\right) \mid(i, j) \in T_{1} \times T_{2}\right\}$, we can replace $\omega^{i^{\prime} p}$ and $\omega^{j^{\prime} p}$ with $\omega^{-i^{\prime}}$ and $\omega^{-j^{\prime}}$, respectively. Therefore, we have

$$
\begin{aligned}
& \left(\omega^{(q-t) j}\right)\left(\omega^{(q-1) i}, \omega^{(q-2) i}, \cdots, \omega^{i}\right) \cdot\left(\omega^{(q-t) j^{\prime} p}\right)\left(\omega^{(q-1) i^{\prime} p}, \omega^{(q-2) i^{\prime} p}, \cdots, \omega^{i^{\prime} p}\right) \\
= & \left(\omega^{(q-t) j}\right)\left(\omega^{(q-1) i}, \omega^{(q-2) i}, \cdots, \omega^{i}\right) \cdot\left(\omega^{(q-t)-j^{\prime}}\right)\left(\omega^{(q-1)-i^{\prime}}, \omega^{(q-2)-i^{\prime}}, \cdots, \omega^{-i^{\prime}}\right) \\
= & \left(\omega^{(q-t)\left(j-j^{\prime}\right)}\right) \sum_{s=1}^{q} \omega^{(q-s)\left(i-i^{\prime}\right)}= \begin{cases}0 & i \neq i^{\prime} \\
q\left(\omega^{(q-t)\left(j-j^{\prime}\right)}\right) & i=i^{\prime}\end{cases}
\end{aligned}
$$


So if $i \neq i^{\prime},<W_{i, j}, W_{i^{\prime}, j^{\prime}}>=0$. Finally, if $i=i^{\prime}$ we have

$$
<W_{i, j}, W_{i^{\prime}, j^{\prime}}>=\sum_{k=1}^{q} \omega^{(q-k)\left(j-j^{\prime}\right)}=\left\{\begin{array}{ll}
0 & j \neq j^{\prime} \\
q^{2} & j=j^{\prime}
\end{array} .\right.
$$

Again if $j \neq j^{\prime},<W_{i, j}, W_{i^{\prime}, j^{\prime}}>=0$. Otherwise, $<W_{i, j}, W_{i^{\prime}, j^{\prime}}>=q^{2}$ and consequently

$$
<b_{i, j}, b_{i^{\prime}, j^{\prime}}>=h . h^{\prime}<W_{i, j}, W_{i^{\prime}, j^{\prime}}>=\left\{\begin{array}{ll}
0 & i \neq i^{\prime} \& j \neq j^{\prime} \\
h . h^{\prime} q^{2}=n_{1} . n_{2} & i=i^{\prime} \& j=j^{\prime}
\end{array} .\right.
$$

In the case $i=i^{\prime}$ and $j=j^{\prime}, n_{1} n_{2}$ is a non zero element and if we multiply each $b_{i, j}$ by a proper constant we can change the norm to 1 .

Second step: Let $s=\operatorname{dim}\left(C \cap C^{\perp_{h}}\right), \beta$ be a $\left(p^{2}-1\right)=(4 k)$ th root of unity, and $G$ be the matrix

$$
G=\left[\begin{array}{cc}
M_{s \times\left(n_{1} \times n_{2}\right)} & 0_{s \times e} \\
A_{\left(\left(n_{1} \times n_{2}\right)-e-2 s\right) \times\left(n_{1} \times n_{2}\right)} & 0_{\left(\left(n_{1} \times n_{2}\right)-e-2 s\right) \times e} \\
B_{e \times\left(n_{1} \times n_{2}\right)} & \beta^{\frac{p-1}{4}} I_{e \times e}
\end{array}\right],
$$

where the rows of $M$ are basis for $C \cap C^{\perp_{h}}$, rows of $A$ and $M$ are basis for $C$, and the set of rows of $B$ is a subset of codewords corresponding to $T$. Moreover, the union of rows of $B$ and $M$ is a basis for $C^{\perp_{h}}$. Let $E$ be the linear code generated by the matrix $G$. By the first step of the proof, one can easily see that all the rows of matrix

$$
S=\left[\begin{array}{cc}
M_{s \times\left(n_{1} \times n_{2}\right)} & 0_{s \times e} \\
B_{e \times\left(n_{1} \times n_{2}\right)} & \beta^{\frac{p-1}{4}} I_{e \times e}
\end{array}\right]
$$

are orthogonal to each row of $G$. For example, if $b_{i}$ and $b_{j}$ are rows in matrix $B$, then the Hermitian inner product is

$$
<b_{i}, b_{j}>=\sum b_{i, k} . \overline{b_{j, k}}=\left\{\begin{array}{ll}
0 & i \neq j \\
1 & i=j
\end{array} .\right.
$$

Now since $\beta^{\frac{p-1}{4}} \cdot \overline{\beta^{\frac{p-1}{4}}}=\beta^{\frac{p-1}{4}} \cdot \beta^{\frac{p(p-1)}{4}}=\beta^{\frac{p^{2}-1}{4}}=-1$, we can guarantee the orthogonality of the last $e$ rows of $S$. Therefore, we are left to show the claim on the minimum distance.

Suppose that $x$ is a codeword generated by the matrix $G$. We have 3 cases:

1. If no row from $B$ occurs in the linear combination of $x$, then $w t(x) \geq d(C)$.

2. If exactly one row of $B$, for example $b_{i, j}$, is in the linear combination with a non-zero coefficient, then $w t(x) \geq d\left(\operatorname{Span}\left(C, b_{i, j}\right)\right)$ and $\operatorname{Span}\left(C, b_{i, j}\right)$ is exactly the 2-D cyclic code with the zero set $U \backslash\left\{\left(\alpha_{1}^{\frac{i n_{1}}{p^{2}-1}}, \alpha_{2} \frac{j n_{2}}{p^{2}-1}\right)\right\}$.

3. Finally, if more than one row of $B$ occur in the linear combination, we can extend case 2 in order to find the appropriate result. 


\subsection{General Quantum Stabilizer Codes Construction Using Classical Additive Codes}

In this Section, we introduce a new method of constructing additive quantum codes, which is a generalization of Theorems 2.3.11 and 2.4.17.

Lemma 3.3.5 Let $C$ be an $[n, l]$ additive code over $\mathbb{F}_{4}$ such that $\operatorname{dim}(C)-\operatorname{dim}(C \cap$ $\left.C^{\perp_{s}}\right)=2 k+i$, where $i \in \mathbb{Z}_{2}$. Then, we can extend $C$ to a new code $Q$, where $Q$ is an $[n+k, l]$ self-orthogonal $\left(Q \subseteq Q^{\perp_{s}}\right)$ additive code over $\mathbb{F}_{4}$.

Proof: Case 1: $i=0$. Suppose $C$ is an additive code with the mentioned properties and $\operatorname{dim}(C)-\operatorname{dim}\left(C \cap C^{\perp_{s}}\right)=2 k$. Let $M$ be the matrix generator of the code $C$, where the last $2 k$ rows are in $C \backslash C \cap C^{\perp_{s}}$ and other rows form a basis for $C \cap C^{\perp_{s}}$. We denote the last $2 k$ rows of $M$ with $m_{1}, m_{2}, \cdots, m_{2 k}$. Since $m_{1} \notin C \cap C^{\perp_{s}}$, there exists at least one $m_{i}, 2 \leq i \leq 2 k$ such that $m_{1} * m_{i}=1$. Without loss of generality, let us assume that $m_{1} * m_{2}=1$. If there is another $m_{j}$ where $3 \leq j \leq 2 k$ such that $m_{1} * m_{j}=1$, then we change $m_{j}$ with $m_{j}+m_{2}$. Moreover, if there exists $m_{z}$ where $3 \leq z \leq 2 k$ such that $m_{z} * m_{2}=1$, we change $m_{z}$ with $m_{z}+m_{1}$. Now, by considering these changes we can find a new format for the last $2 k$ rows of $M$, such as $m_{1}, m_{2}, m_{1}^{\prime}, m_{2}^{\prime}, \cdots, m_{2 k-2}^{\prime}$ such that $m_{1} * m_{2}=1$ and for any $m_{t}^{\prime}$ where $1 \leq t \leq 2 k-2$ we have $m_{t}^{\prime} * m_{1}=m_{t}^{\prime} * m_{2}=0$. Now, we repeat the above method on $m_{1}^{\prime}, m_{2}^{\prime}, \cdots, m_{2 k-2}^{\prime}$. By continuing this procedure we can find vectors $M_{1}, M_{2}, \cdots, M_{2 k}$ in the last $2 k$ rows such that $M_{2 i-1} * M_{2 i}=1$ and $M_{2 i-1} * M_{p}=M_{2 i} * M_{q}=0$ for $1 \leq i \leq k, p \neq 2 i$, and $q \neq 2 i-1$.

Now, let $T$ be a matrix such that $T_{2 s-1, s}=1$ and $T_{2 s, s}=\omega$ for $1 \leq s \leq k$, and the other entries of $T$ are zero. The matrix

$$
G=\left[\begin{array}{ll}
M_{s \times n} & 0_{s \times k} \\
A_{2 k \times n} & T_{2 k \times k}
\end{array}\right],
$$

where $s=\operatorname{dim}\left(C \cap C^{\perp_{s}}\right)$ is the generator matrix of an $[n+k, l]$ additive code $Q$ over $\mathbb{F}_{4}$ and one can easily see that $Q \subseteq Q^{\perp_{s}}$.

Case 2: $i=1$. Let us assume that $\operatorname{dim}(C)-\operatorname{dim}\left(C \cap C^{\perp_{s}}\right)=2 k+1$. Using similar steps, we are able to find the vectors $M_{1}, M_{2}, \cdots, M_{2 k}, M_{2 k+1}$ for the last $2 k+1$ rows such that $M_{2 i-1} * M_{2 i}=1$ and $M_{2 i-1} * M_{p}=M_{2 i} * M_{q}=0$ for $1 \leq i \leq k, p \neq 2 i$, and $q \neq 2 i-1$, and $M_{2 k+1}$ is orthogonal to all other vectors. In this case, let $T^{\prime}$ be the the vertical join of matrix $T$ and one zero row. Now, the matrix

$$
G=\left[\begin{array}{cc}
M_{s \times n} & 0_{s \times k} \\
A_{2 k+1 \times n} & T_{2 k+1 \times k}^{\prime}
\end{array}\right]
$$

is the generator matrix of the code $Q$ and clearly $Q \subseteq Q^{\perp_{s}}$.

Now, we state our main result.

Theorem 3.3.6 Let $C$ be an $[n, k]$ additive code over $\mathbb{F}_{4}$ and $e=\left[\frac{2 n-k-\left(\operatorname{dim}\left(C \cap C^{\perp s}\right)\right)}{2}\right]$. If $2 n-k-\left(\operatorname{dim}\left(C \cap C^{\perp_{s}}\right)\right)$ is an odd integer, then there exists an $[[n+e, k-n+e+1, d]]$ quantum code over $\mathbb{F}_{4}$ with $d \geq \min \left\{d(C+v),\left(d\left(C+C^{\perp_{s}}\right)+1\right)\right\}$ for all $v \neq 0 \in C^{\perp_{s}}$. If $2 n-k-\left(\operatorname{dim}\left(C \cap C^{\perp_{s}}\right)\right)$ is an even integer, then there exists an $[[n+e, k-n+e, d]]$ quantum code over $\mathbb{F}_{4}$ with $d \geq \min \left\{d(C),\left(d\left(C+C^{\perp_{s}}\right)+1\right)\right\}$. 
Proof: Let $C$ be an additive code with dimension $k$ and $C^{\perp_{s}}$ be its dual with respect to the symplectic inner product $\left(\operatorname{dim}\left(C^{\perp_{s}}\right)=2 n-k\right.$ over $\left.\mathbb{F}_{2}\right)$. Let $e=\left[\frac{2 n-k-\left(\operatorname{dim}\left(C \cap C^{\perp s}\right)\right)}{2}\right]$, and $\operatorname{dim}\left(C \cap C^{\perp_{s}}\right)=s$. We consider two cases.

Case 1. Assume that $2 n-k-\left(\operatorname{dim}\left(C \cap C^{\perp_{t}}\right)\right)$ is an odd integer. Consider the matrix

$$
G=\left[\begin{array}{cc}
M_{s \times n} & 0_{s \times e} \\
A_{k-s \times n} & 0_{k-s \times e} \\
B_{2 e \times n} & T_{2 e \times e} \\
v & 0_{1 \times e}
\end{array}\right],
$$

where $r(M)$ is a basis for $C \cap C^{\perp_{s}}, r(A) \cup r(M)$ is a basis for $C, r(B)=M_{1}, M_{2}, \cdots, M_{2 e}$, and $v=M_{2 e+1}$, where $M_{i}$ 's satisfy in second part of the proof of Lemma 3.3.5. Finally, the set $r(B) \cup\{v\} \cup r(M)$ is a basis for $C^{\perp_{s}}$, and $T$ is the matrix which was introduced in the proof of Lemma 3.3.5. Let $E$ be the additive code generated by the matrix $G$. Now, consider the code generated by

$$
S=\left[\begin{array}{ll}
M_{s \times n} & 0_{s \times e} \\
B_{2 e \times n} & T_{2 e \times e}
\end{array}\right] .
$$

By the construction and the above lemma, rows of $S$ are orthogonal to the rows of $G$. Moreover, $\operatorname{dim}(E)=2 n-s$ and $\operatorname{dim}\left(E^{\perp_{s}}\right)=s+2 e$. Therefore, $S$ is the generator matrix of the code $E^{\perp_{s}}$ and $E^{\perp_{s}} \subseteq E$.

Assume $x=\left(x_{1}, x_{2}\right) \in E$ where $x_{1} \in \mathbb{F}_{4}^{n}$ and $x_{2} \in \mathbb{F}_{4}^{e}$. So $x$ is linear combination of rows of $G$. If no row of $B$ appears in the linear combination, then $w t(x) \geq d(C+v)$. If some of the rows of $B$ enter this linear combination $w t(x) \geq d\left(\left(C+C^{\perp_{s}}\right)+1\right.$. Case 2. Assume that $2 n-k-\left(\operatorname{dim}\left(C \cap C^{\perp_{s}}\right)\right)$ is even. then

$$
G=\left[\begin{array}{cc}
M_{s \times n} & 0_{s \times e} \\
A_{k-s \times n} & 0_{k-s \times e} \\
B_{2 e \times n} & T_{2 e \times e}
\end{array}\right]
$$

is the matrix generator of the code $E$ and its dual is generated by the matrix $S$. Rest of the proof is similar to the case 1 .

Using Theorem 3.3.6 we are able to construct a quantum code from an additive code. Note that this construction can easily be extended to $\mathbb{F}_{q}$ additive codes over $\mathbb{F}_{q^{2}}$.

Corollary 3.3.7 Let $C$ be an $[n, k]_{q}$ additive code over $\mathbb{F}_{q^{2}}$ and $e=\left[\frac{2 n-k-\left(\operatorname{dim}\left(C \cap C^{\perp s}\right)\right)}{2}\right]$. If $2 n-k-\left(\operatorname{dim}\left(C \cap C^{\perp_{s}}\right)\right)$ is an odd integer, then there exists an $[[n+e, k-n+e+1, d]]_{q}$ quantum code over $\mathbb{F}_{q^{2}}$ with $d \geq \min \left\{d(C+v),\left(d\left(C+C^{\perp_{s}}\right)+1\right)\right\}$ for some $v \neq 0 \in C^{\perp_{s}}$.

If $2 n-k-\left(\operatorname{dim}\left(C \cap C^{\perp_{s}}\right)\right)$ is an even integer, then there exists an $[[n+e, k-n+e, d]]_{q}$ quantum code over $\mathbb{F}_{q^{2}}$ with $d \geq \min \left\{d(C),\left(d\left(C+C^{\perp_{s}}\right)+1\right)\right\}$.

Corollary 3.3.8 Let $C$ be an $[n, k]_{q}$ linear code over $\mathbb{F}_{q^{2}}$ and $e^{\prime}=n-k-(\operatorname{dim}(C \cap$ $\left.\left.C^{\perp_{s}}\right)\right)$. Then there exists an $\left[\left[n+e^{\prime}, 2 k-n+e^{\prime}, d\right]\right]$ quantum code with $d \geq \min \{d(C),(d(C+$ $\left.\left.\left.C^{\perp_{s}}\right)+1\right)\right\}$. 
Proof: Let $C$ and $C \cap C^{\perp_{s}}$ be $[n, k]$ and $[n, s]$ linear codes over $\mathbb{F}_{q^{2}}$, respectively. We can consider them as $[n, 2 k]$ and $[n, 2 s]$ additive code over $\mathbb{F}_{q^{2}}$. Now, by Corollary 3.3.7 we have

$$
e=\left[\frac{2 n-k-\left(\operatorname{dim}\left(C \cap C^{\perp s}\right)\right)}{2}\right]=\left[\frac{2 n-2 k-2 s}{2}\right]=n-k-s=e^{\prime},
$$

and there exists an $\left[\left[n+e^{\prime}, 2 k-n+e^{\prime}, d\right]\right]$ quantum code with $d \geq \min \{d(C),(d(C+$ $\left.\left.\left.C^{\perp_{s}}\right)+1\right)\right\}$.

Example 3.3.9 In this Example we explain how to use Lemma 3.3.5 and Theorem 3.3.6 to obtain a quantum stabilizer code with a good minimum distance. Let us start with an $[33,16,11]$ linear code $C$ which is the best known linear code over $\mathbb{F}_{4}$ with these parameters.

Using Magma, we see that $C \cap C^{\perp_{s}}$ is a $[33,15]$ linear code which means that $e=1$. By Theorem 3.3.6 we can obtain a $[[34,2,10]]$ quantum code which has the optimum minimum distance (see [8]).

Example 3.3.10 The following table presents some good quantum codes obtained from Theorem 3.3.6.

\begin{tabular}{|l||l|}
\hline \multicolumn{2}{|c|}{ Parameters of the codes } \\
\hline Codes over $\mathbb{F}_{4}$ & Codes over $\mathbb{F}_{9}$ \\
\hline$[[34,2,10]]$ & {$[[10,1,4]]$} \\
{$[[35,1,11]]$} & {$[[11,1,5]]$} \\
{$[[46,8,9]]$} & {$[[28,22,3]]$} \\
{$[[47,7,10]]$} & {$[[30,22,3]]$} \\
{$[[51,3,13]]$} & {$[[32,20,4]]$} \\
{$[[52,2,14]]$} & {$[[32,22,4]]$} \\
{$[[53,1,15]]$} & {$[[34,18,6]]$} \\
{$[[58,4,14]]$} & {$[[41,25,6]]$} \\
{$[[61,1,17]]$} & {$[[41,33,4]]$} \\
\hline
\end{tabular}




\section{Bibliography}

[1] Ashikhmin A, Knill E. Nonbinary quantum stabilizer codes. IEEE Transactions on Information Theory. 2001 Nov;47(7):3065-72.

[2] Calderbank AR, Shor PW. Good quantum error-correcting codes exist. Physical Review A. 1996 Aug 1;54(2):1098.

[3] Calderbank AR, Rains EM, Shor PM, Sloane NJ. Quantum error correction via codes over GF (4). IEEE Transactions on Information Theory. 1998 Jul;44(4):136987.

[4] Güneri C. Artin-Schreier curves and weights of two-dimensional cyclic codes. Finite Fields and Their Applications. 2004 Oct 1;10(4):481-505.

[5] Degwekar A, Guenda K, Gulliver TA. Extending construction X for quantum error-correcting codes. In Coding Theory and Applications 2015 (pp. 141-152). Springer International Publishing.

[6] Dieks DG. Communication by EPR devices. Physics Letters A. 1982 Nov $22 ; 92(6): 271-2$.

[7] Gottesman D. Class of quantum error-correcting codes saturating the quantum Hamming bound. Physical Review A. 1996 Sep 1;54(3):1862.

[8] Grassl M. Bounds on the minimum distance of linear codes and quantum codes (2007). Online available at http://www.codetables.de. Accessed on 2017-04-30.

[9] Imai H. A theory of two-dimensional cyclic codes. Information and Control. 1977 May 1;34(1):1-21.

[10] Lisonk P, Singh V. Quantum codes from nearly self-orthogonal quaternary linear codes. Designs, Codes and Cryptography. 2014 Nov 1;73(2):417-24.

[11] Sepasdar Z. Some notes on the characterization of two dimensional skew cyclic codes. Journal of Algebra and Related Topics. 2016 Dec 20;4(2):1-8.

[12] Shor PW. Scheme for reducing decoherence in quantum computer memory. Physical review A. 1995 Oct 1;52(4):R2493.

[13] MacWilliams FJ, Sloane NJ. The Theory of Error-Correcting Codes. Elsevier; 1977.

[14] Steane AM. Simple quantum error-correcting codes. Physical Review A. 1996 Dec $1 ; 54(6): 4741$. 
[15] Steane A. Multiple-particle interference and quantum error correction. InProceedings of the Royal Society of London A: Mathematical, Physical and Engineering Sciences 1996 Nov 8 (Vol. 452, No. 1954, pp. 2551-2577). The Royal Society.

[16] Wootters WK, Zurek WH. A single quantum cannot be cloned. Nature. 1982 Oct 28;299(5886):802-3. 